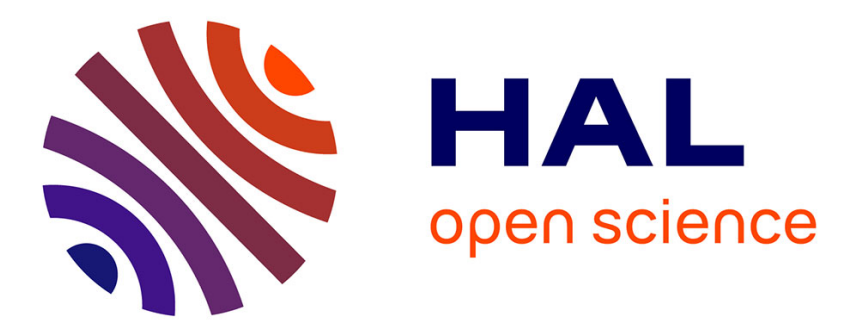

\title{
Approximation by quantization of the filter process and applications to optimal stopping problems under partial observation
}

Huyen Pham, Wolfgang Runggaldier, Afef Sellami

\section{- To cite this version:}

Huyen Pham, Wolfgang Runggaldier, Afef Sellami. Approximation by quantization of the filter process and applications to optimal stopping problems under partial observation. 2004. hal-00002973

\section{HAL Id: hal-00002973 \\ https://hal.science/hal-00002973}

Preprint submitted on 29 Sep 2004

HAL is a multi-disciplinary open access archive for the deposit and dissemination of scientific research documents, whether they are published or not. The documents may come from teaching and research institutions in France or abroad, or from public or private research centers.
L'archive ouverte pluridisciplinaire HAL, est destinée au dépôt et à la diffusion de documents scientifiques de niveau recherche, publiés ou non, émanant des établissements d'enseignement et de recherche français ou étrangers, des laboratoires publics ou privés. 
Universités de Paris 6 \& Paris 7 - CNRS (UMR 7599)

\section{PRÉPUBLICATIONS DU LABORATOIRE}

DE PROBABILITÉS \& MODÈLES ALÉATOIRES

4, place Jussieu - Case 188 - 75252 Paris cedex 05 http://www.proba.jussieu.fr 


\begin{abstract}
Approximation by quantization of the filter process and applications to optimal stopping problems under partial observation
\end{abstract}

H. PHAM, W. RUNGGALDIER \& A. SELLAMI

\title{
SEPTEMBRE 2004
}

Prépublication $n^{\circ} 925$

H. Pham : Laboratoire de Probabilités et Modèles Aléatoires, CNRS-UMR 7599, Université Paris VI \& Université Paris VII, 4 place Jussieu, Case 188, F-75252 Paris Cedex $05 \&$ CREST.

W. Runggaldier : Dipartimento di Matematica Pura ed Applicata, Universit degli Studi di Padova, Via Belzoni 7, 35100 Padova, Italy.

A. Sellami : Laboratoire de Probabilités et Modèles Aléatoires, CNRS-UMR 7599, Université Paris VI \& Université Paris VII, 4 place Jussieu, Case 188, F-75252 Paris Cedex 05. 


\title{
Approximation by quantization of the filter process and applications to optimal stopping problems under partial observation
}

\author{
Huyên PHAM* \\ Laboratoire de Probabilités et \\ Modèles Aléatoires \\ CNRS, UMR 7599 \\ Université Paris 7 \\ pham@math.jussieu.fr \\ and CREST
}

\author{
Wolfgang RUNGGALDIER \\ Dipartimenta di Matematica \\ Pura ed Applicata \\ Universita degli studi di Padova \\ runggal@math.unipd.it
}

\author{
Afef SELLAMI \\ Laboratoire de Probabilités et \\ Modèles Aléatoires \\ CNRS, UMR 7599 \\ Université Paris 7 \\ sellami@math.jussieu.fr
}

\begin{abstract}
We present an approximation method for discrete time nonlinear filtering in view of solving dynamic optimization problems under partial information. The method is based on quantization of the Markov pair process filter-observation $(\Pi, Y)$ and is such that, at each time step $k$ and for a given size $N_{k}$ of the quantization grid in period $k$, this grid is chosen to minimize a suitable quantization error. The algorithm is based on a stochastic gradient descent combined with Monte-Carlo simulations of $(\Pi, Y)$. Convergence results are given and applications to optimal stopping under partial observation are discussed. Numerical results are presented for a particular stopping problem : American option pricing with unobservable volatility.
\end{abstract}

Key words: Nonlinear filtering, Markov chain, quantization, stochastic gradient descent, Monte-Carlo simulations, partial observation, optimal stopping.

MSC Classification (2000): 60G35, 65C20, 65N50, 62L20, 60G40.

*Corresponding author 


\section{Introduction}

We consider a discrete time, partially observable process $(X, Y)$ where $X$ represents the state or signal process that may not be observable, while $Y$ is the observation. The signal process $\left\{X_{k}, k \in \mathbb{N}\right\}$ is valued in a measurable space $(E, \mathcal{E})$ and is a Markov chain with probability transition $\left(P_{k}\right)$ (i.e. the transition from time $k-1$ to time $k$ ), and initial law $\mu$. The observation sequence $\left(Y_{k}\right)$ is valued in $\mathbb{R}^{d}$ and such that the pair $\left(X_{k}, Y_{k}\right)$ is a Markov chain and

(H) The law of $Y_{k}$ conditional on $\left(X_{k-1}, Y_{k-1}, X_{k}\right), k \geq 1$, denoted $q_{k}\left(X_{k-1}, Y_{k-1}, X_{k}, d y^{\prime}\right)$, admits a bounded density

$$
y^{\prime} \longmapsto g_{k}\left(X_{k-1}, Y_{k-1}, X_{k}, y^{\prime}\right) .
$$

For simplicity, we assume that $Y_{0}$ is a known deterministic constant equal to $y_{0}$. Notice that the probability transition of the Markov chain $\left(X_{k}, Y_{k}\right)_{k \in \mathbb{N}}$ is then given by $P_{k}\left(x, d x^{\prime}\right) g_{k}\left(x, y, x^{\prime}, y^{\prime}\right) d y^{\prime}$ with initial law $\mu(d x) \delta_{y_{0}}(d y)$.

We denote by $\left(\mathcal{F}_{k}^{Y}\right)$ the filtration generated by the observation process $\left(Y_{k}\right)$ and by $\Pi_{k}$ the filter conditional law of $X_{k}$ given $\mathcal{F}_{k}^{Y}$ :

$$
\Pi_{k}(d x)=\mathbb{P}\left[X_{k} \in d x \mid \mathcal{F}_{k}^{Y}\right], \quad k \in \mathbb{N} .
$$

The filter process $\left(\Pi_{k}\right)_{k}$ allows one to transform problems related to the partially observed process $(X, Y)$ into equivalent problems under complete observation related to the pair $(\Pi, Y)$ and this latter pair turns out to be Markov with respect to the observation filtration $\left(\mathcal{F}_{k}^{Y}\right)$. An important class of problems related to partially observable processes are stochastic control and stopping problems that are dynamic stochastic optimization problems where the partial observation concerns the state/signal process. We shall, in particular, consider optimal stopping under partial information and, in this context, a financial application concerning the pricing of American options in a partially observed stochastic volatility model.

We shall assume that the state space $E$ of the state/signal process $\left(X_{k}\right)$ consists of a finite number $m$ of points. Problems with a more general state space can be approximated by problems with a finite state space (see e.g. [8]). With $X_{k}$ taking the $m$ values $x^{i}(i=$ $1, \cdots, m)$, the filter process is characterized by an $m$-vector with components $\Pi_{k}^{i}=\mathbb{P}\left[X_{k}=\right.$ $\left.x^{i} \mid \mathcal{F}_{k}^{Y}\right]$ and takes thus values in the $m$-simplex $K_{m}$ in $\mathbb{R}^{m}$. While the filter process allows thus to transform a problem under partial observation into one under full observation, it has the drawback that a finite-valued state space becomes infinite. This leads to difficulties when trying to solve dynamic optimization problems also in discrete time. For actual computation one has thus to discretize the process $\left(\Pi_{k}\right)$, approximating it with a process that takes a finite number of values in the simplex $K_{m}$. Approaches to this effect have appeared in the literature; they are based on discretizing the observations process $\left(Y_{k}\right)$ and then approximating $\Pi_{k}$ by a filter of $X_{k}$, given the discretized observations path (see e.g. $[5],[3],[15],[10]$ and references therein). Such an approach has the drawback that the number of possible values for the approximating filter grows exponentially fast with the time step. 
In this paper, we propose a new approach by exploiting the Markov property of $(\Pi, Y)_{k}$ with respect to the observation filtration $\left(\mathcal{F}_{k}^{Y}\right):$ the conditional law of $X_{k+1}$ given $\mathcal{F}_{k}^{Y}$ is summarized by the sufficient statistics $\left(\Pi_{k}, Y_{k}\right)$ valued in $K^{m} \times \mathbb{R}^{d}$. This suggests to approximate the couple process $(\Pi, Y)$ with $(\hat{\Pi}, \hat{Y})$ that in the generic time step $k$ takes a number of values $N_{k}$ that can be assigned arbitrarily. Following standard usage, we shall call this quantization and the problem then arises to find an optimal quantization, namely such that it minimizes for each time period $k$ the $L^{2}$-approximation error induced by the quantization. This error is related to what e.g. in information theory is called distorsion. The implementation of the minimizing algorithm itself is based on a stochastic gradient descent method combined with Monte-Carlo simulations of (,$Y)$. As a byproduct, we obtain an approximation of the probability transition matrices of the Markov chain $(\Pi, Y)$. These companion parameters provide in turn an approximation algorithm, via the dynamic programming principle, for computing optimal values associated to dynamic optimization problems under partial observation.

Optimal quantization methods have been developed recently in numerical probability and various problems of optimal stopping, control or nonlinear filtering, see [11], [1], [2], [13], [14], [12]. In our context, we obtain error bounds and rate of convergence for the approximation of the pair filter-observation process $(\Pi, Y)$. We then give an application to the approximation of optimal stopping problem under partial observation and study associated convergence results. Finally, we present a numerical illustration for the American option pricing problem with unobservable volatility.

The rest of the paper is organized as follows. In Section 2, we recall some preliminaries on nonlinear filtering. Section 3 is the heart of the paper. We first present the quantization approximation method for the filter-observation Markov chain, and then analyze the induced error. The rate of convergence and the practical implementation of the algorithm for the optimal quantization are discussed. In Section 4, we apply our quantization method to the approximation of an optimal stopping problem under partial observation. Convergence results for the associated optimal value functions are provided. The last Section 5 presents numerical tests for the American option pricing with unobservable volatility.

\section{Preliminaries}

We denote by $\mathcal{M}(E)$ the set of finite nonnegative measures on $(E, \mathcal{E})$ and by $\mathcal{P}(E)$ the subset of probability measures on $(E, \mathcal{E})$. It is known that $\mathcal{M}(E)$ is a Polish space equipped with the weak topology, hence a measurable space endowed with the Borel $\sigma$-field. We recall that from Bayes formula, $\Pi_{k}$ is given in inductive form by :

$$
\begin{aligned}
& \Pi_{0}=\mu \\
& \Pi_{k}=\bar{G}_{k}\left(\Pi_{k-1}, Y_{k-1}, Y_{k}\right), \quad k \geq 1,
\end{aligned}
$$

where $G_{k}$ is the continuous function from $\mathcal{M}(E) \times \mathbb{R}^{d} \times \mathbb{R}^{d}$ into $\mathcal{M}(E)$ defined by :

$$
G_{k}\left(\pi, y, y^{\prime}\right)\left(d x^{\prime}\right)=\int_{E} g_{k}\left(x, y, x^{\prime}, y^{\prime}\right) P_{k}\left(x, d x^{\prime}\right) \pi(d x)
$$


and $\bar{G}_{k}$ is the normalized continuous function valued in $\mathcal{P}(E)$ :

$$
\bar{G}_{k}\left(\pi, y, y^{\prime}\right)=\frac{G_{k}\left(\pi, y, y^{\prime}\right)}{\int_{E} G_{k}\left(\pi, y, y^{\prime}\right)\left(d x^{\prime}\right)}
$$

Denoting by $\left(\mathcal{F}_{k}\right)$ the filtration generated by $\left(X_{k}, Y_{k}\right)$, and using the law of iterated conditional expectations, we have for any $k$ and bounded Borelian function $\varphi$ on $\mathcal{P}(E) \times \mathbb{R}^{d}$ :

$$
\begin{aligned}
& \mathbb{E}\left[\varphi\left(\Pi_{k+1}, Y_{k+1}\right) \mid \mathcal{F}_{k}^{Y}\right] \\
= & \mathbb{E}\left[\mathbb{E}\left[\varphi\left(\bar{G}_{k+1}\left(\Pi_{k}, Y_{k}, Y_{k+1}\right), Y_{k+1}\right) \mid \mathcal{F}_{k}\right] \mid \mathcal{F}_{k}^{Y}\right] \\
= & \mathbb{E}\left[\int \varphi\left(\bar{G}_{k+1}\left(\Pi_{k}, Y_{k}, y^{\prime}\right), y^{\prime}\right) P_{k+1}\left(X_{k}, d x^{\prime}\right) q_{k+1}\left(X_{k}, Y_{k}, x^{\prime}, d y^{\prime}\right) \mid \mathcal{F}_{k}^{Y}\right] \\
= & \int \varphi\left(\bar{G}_{k+1}\left(\Pi_{k}, Y_{k}, y^{\prime}\right), y^{\prime}\right) P_{k+1}\left(x, d x^{\prime}\right) q_{k+1}\left(x, Y_{k}, x^{\prime}, d y^{\prime}\right) \Pi_{k}(d x) .
\end{aligned}
$$

This proves that the pair $\left(\Pi_{k}, Y_{k}\right)_{k}$ is a $\left(\mathbb{P},\left(\mathcal{F}_{k}^{Y}\right)_{k}\right)$ Markov chain in $\mathcal{P}(E) \times \mathbb{R}^{d}$, with initial value $\left(\mu, y_{0}\right)$. Moreover, under $(\mathbf{H})$, this also shows that the (unnormalized) law of $Y_{k}$ conditional on $\left(\Pi_{k-1}, Y_{k-1}\right)$, denoted $Q_{k}\left(\Pi_{k-1}, Y_{k-1}, d y^{\prime}\right)$ admits a density given by :

$$
y^{\prime} \longmapsto \int G_{k}\left(\Pi_{k-1}, Y_{k-1}, y^{\prime}\right)\left(d x^{\prime}\right)=\int g_{k}\left(x, Y_{k-1}, x^{\prime}, y^{\prime}\right) P_{k}\left(x, d x^{\prime}\right) \Pi_{k-1}(d x) .
$$

Notice that the probability transition $R_{k}$ (from time $k-1$ to $k$ ) of the Markov chain $\left(\Pi_{k}, Y_{k}\right)_{k}$ is not explicit in general. Actually, from (2.2), we can write :

$$
R_{k} \varphi(\pi, y)=\int \varphi\left(\bar{G}_{k}\left(\pi, y, y^{\prime}\right), y^{\prime}\right) Q_{k}\left(\pi, y, d y^{\prime}\right), \quad \forall(\pi, y) \in \mathcal{P}(E) \times \mathbb{R}^{d} .
$$

In the sequel, we denote by $|\cdot|_{2}$ the Euclidian norm and by $|\cdot|_{1}$ the $l^{1}$ norm on $\mathbb{R}^{l}$. For any $\mathbb{R}^{l}$-valued random variable $U$, we denote :

$$
\|U\|_{2}=\left(\mathbb{E}|U|_{2}^{2}\right)^{\frac{1}{2}} \text { and }\|U\|_{1}=\mathbb{E}|U|_{1}
$$

\section{An optimal quantization approach for the approximation of the filter process}

\subsection{The approximation method}

We assume here that the state space $E$ of the signal process $\left(X_{k}\right)$ is finite consisting of $m$ points : $E=\left\{x^{1}, \ldots, x^{m}\right\}$. The initial discrete law $\mu=\left(\mu^{i}\right)$ and the probability transition matrix $P_{k}$ are defined by :

$$
\begin{aligned}
\mu^{i} & =\mathbb{P}\left[X_{0}=x^{i}\right], \quad i=1, \ldots, m, \\
P_{k}^{i j} & =\mathbb{P}\left[X_{k}=x^{j} \mid X_{k-1}=x^{i}\right], \quad i, j=1, \ldots, m .
\end{aligned}
$$

The random filter $\Pi_{k}$ is characterized by its random weights :

$$
\Pi_{k}^{i}=\mathbb{P}\left[X_{k}=x^{i} \mid \mathcal{F}_{k}^{Y}\right], \quad i=1, \ldots, m,
$$


and may then be identified with a random vector valued in the $m$-simplex $K_{m}$ in $\mathbb{R}^{m}$ of dimension $m-1$ :

$$
K_{m}=\left\{\pi=\left(\pi^{i}\right) \in \mathbb{R}^{m}: \pi^{i} \geq 0,1 \leq i \leq m,|\pi|_{1}=\sum_{i=1}^{m} \pi^{i}=1\right\} .
$$

By (2.1), it is expressed in the recursive form :

$$
\begin{aligned}
\Pi_{0} & =\mu \\
\Pi_{k} & =\bar{G}_{k}\left(\Pi_{k-1}, Y_{k-1}, Y_{k}\right)=\frac{G P_{k}\left(Y_{k-1}, Y_{k}\right)^{\top} \Pi_{k-1}}{\left|G P_{k}\left(Y_{k-1}, Y_{k}\right)^{\top} \Pi_{k-1}\right|_{1}},
\end{aligned}
$$

where $G P_{k}\left(Y_{k-1}, Y_{k}\right)$ is a $m \times m$ random matrix given by :

$$
G P_{k}\left(Y_{k-1}, Y_{k}\right)_{i j}=g_{k}\left(x_{k-1}^{i}, Y_{k-1}, x_{k}^{j}, Y_{k}\right) P_{k}^{i j}, \quad 1 \leq i, j \leq m .
$$

Here $M^{\top}$ denotes the transpose of a matrix $M$.

A first approach for approximating the filter process $\left(\Pi_{k}\right)$ consists in discretizing the observation process $\left(Y_{k}\right)$ by replacing it by a process $\left(\hat{Y}_{k}\right)$ taking a finite number $N$ of values, and then approximate for each $k$ the random filter $\Pi_{k}$ by a random filter of $X_{k}$ given $\hat{Y}_{1}, \ldots, \hat{Y}_{k}$. So at each time step $k$, given $\hat{Y}_{k}$ and $\hat{\Pi}_{k}$, the random variable $\hat{\Pi}_{k+1}$ may take $N$ values. Thus, at time $n$, the random filter $\hat{\Pi}_{n}$ is identified with a random vector taking $N^{n}$ possible values. All these values are precomputed and stored in a "look-up table" but this could be very heavy, typically when $n$ is large. Such an approach was introduced in [5] and [3], and investigated further in [15],[10] and [16].

We propose here a new approach. This starts from the key remark that the pair process $\left(\Pi_{k}, Y_{k}\right)$ is Markov with respect to the observation filtration $\left(\mathcal{F}_{k}^{Y}\right)$. In other words, the conditional law of $X_{k+1}$ given $\mathcal{F}_{k}^{Y}$ may be summarized by the sufficient statistic $\left(\Pi_{k}, Y_{k}\right)$. Therefore, since the Markov chain $\left(\Pi_{k}, Y_{k}\right)$ is completely characterized by its probability transitions, the idea is to approximate these probability transitions by suitable probability transition matrices.

In a first step, we discretize for each $k$ the couple $\left(\Pi_{k}, Y_{k}\right)$ by approximating it by $\left(\hat{\Pi}_{k}, \hat{Y}_{k}\right)$ taking a finite number of values. The space discretization (or quantization) of the random vector $Z_{k}=\left(\Pi_{k}, Y_{k}\right)$ in $K_{m} \times \mathbb{R}^{d}$ is constructed as follows. At initial time $k=0$, recall that $Z_{0}$ is a known deterministic vector equal to $z_{0}=\left(\mu, y_{0}\right)$, so we start from the grid with one point in $K_{m} \times \mathbb{R}^{d}$ :

$$
\Gamma_{0}=\left\{z_{0}=\left(\mu, y_{0}\right)\right\}
$$

At time $k \geq 1$, we are given a grid $\Gamma_{k}$ of $N_{k}$ points in $K_{m} \times \mathbb{R}^{d}$ :

$$
\Gamma_{k}=\left\{z_{k}^{1}=\left(\pi_{k}(1), y_{k}^{1}\right), \ldots, z_{k}^{N_{k}}=\left(\pi_{k}\left(N_{k}\right), y_{k}^{N_{k}}\right)\right\},
$$

and we denote by $C_{i}\left(\Gamma_{k}\right), i=1, \ldots, N_{k}$, the associated Voronoi tesselations :

$$
C_{i}\left(\Gamma_{k}\right)=\left\{z \in K_{m} \times \mathbb{R}^{d}: \operatorname{Proj}_{\Gamma_{k}}(z)=z_{k}^{i}\right\}, \quad i=1, \ldots, N_{k} .
$$


Here $\operatorname{Proj}_{\Gamma_{k}}$ is a closest neighbor projection for the Euclidian norm :

$$
\left|z-\operatorname{Proj}_{\Gamma_{k}}(z)\right|_{2}=\min _{i=1, \ldots, N_{k}}\left|z-z_{k}^{i}\right|_{2}, \quad \forall z \in K_{m} \times \mathbb{R}^{d} .
$$

Notice that by definition of the Euclidian norm, we clearly have :

$$
\operatorname{Proj}_{\Gamma_{k}}=\left(\operatorname{Proj}_{\Gamma_{k}^{\Pi}}, \operatorname{Proj}_{\Gamma_{k}^{Y}}\right), \quad C_{i}\left(\Gamma_{k}\right)=C_{i}\left(\Gamma_{k}^{\Pi}\right) \times C_{i}\left(\Gamma_{k}^{Y}\right),
$$

where :

$$
\begin{aligned}
\Gamma_{k}^{\Pi} & =\left\{\pi_{k}(1), \ldots, \pi_{k}\left(N_{k}\right)\right\} \\
\Gamma_{k}^{Y} & =\left\{y_{k}^{1}, \ldots, y_{k}^{N_{k}}\right\} .
\end{aligned}
$$

We then approximate the pair $Z_{k}=\left(\Pi_{k}, Y_{k}\right)$ by $\hat{Z}_{k}=\left(\hat{\Pi}_{k}, \hat{Y}_{k}\right)$ valued in $\Gamma_{k}$ and defined by :

$$
\hat{Z}_{k}=\operatorname{Proj}_{\Gamma_{k}}\left(Z_{k}\right)=\left(\operatorname{Proj}_{\Gamma_{k}^{\Pi}}\left(\Pi_{k}\right), \operatorname{Proj}_{\Gamma_{k}^{Y}}\left(Y_{k}\right)\right) .
$$

In a second step, we approximate the probability transitions of the Markov chain $\left(Z_{k}\right)$ :

$$
R_{k}\left(z, d z^{\prime}\right)=\mathbb{P}\left[Z_{k} \in d z^{\prime} \mid Z_{k-1}=z\right], \quad k \geq 1, z \in K_{m} \times \mathbb{R}^{d},
$$

by the following probability transition matrix :

$$
\begin{aligned}
\hat{r}_{k}^{i j} & =\mathbb{P}\left[\hat{Z}_{k}=z_{k}^{j} \mid \hat{Z}_{k-1}=z_{k-1}^{i}\right] \\
& =\frac{\mathbb{P}\left[Z_{k} \in C_{j}\left(\Gamma_{k}\right), Z_{k-1} \in C_{i}\left(\Gamma_{k-1}\right)\right]}{\mathbb{P}\left[Z_{k-1} \in C_{i}\left(\Gamma_{k-1}\right)\right]}=: \frac{\hat{\beta}_{k}^{i j}}{\hat{p}_{k-1}^{i}},
\end{aligned}
$$

for all $k \geq 1, i=1, \ldots, N_{k-1}, j=1, \ldots, N_{k}$. We shall see later how the grids $\Gamma_{k}$ and the number of points $N_{k}$ are optimally chosen and implemented, and how the associated probability transition matrix $\hat{r}_{k}$ can be estimated.

Example : Computation of predictor conditional expectation.

Suppose we are interested in the computation for any $k=0, \ldots, n$, and for arbitrary measurable function $\varphi_{k+1}$ on $K_{m} \times \mathbb{R}^{d}$, of the filter predictor :

$$
U_{k}=\mathbb{E}\left[\varphi_{k+1}\left(X_{k+1}, Y_{k+1}\right) \mid \mathcal{F}_{k}^{Y}\right] .
$$

A precise application where such $\mathcal{F}_{k}^{Y}$-measurable random variables appear is presented in Section 4 . Then, by introducing the function :

$$
\hat{\varphi}_{k+1}(\pi, y)=\sum_{i=1}^{m} \varphi_{k+1}\left(x^{i}, y\right) \pi^{i}, \quad \forall \pi=\left(\pi^{i}\right)_{i} \in K_{m}, \forall y \in \mathbb{R}^{d},
$$

and using the law of iterated conditional expectation, we can rewrite $U_{k}$ as :

$$
U_{k}=\mathbb{E}\left[\hat{\varphi}_{k+1}\left(\Pi_{k+1}, Y_{k+1}\right) \mid \mathcal{F}_{k}^{Y}\right]=\mathbb{E}\left[\hat{\varphi}_{k+1}\left(Z_{k+1}\right) \mid \mathcal{F}_{k}^{Y}\right] .
$$

We thus approximate the sequence of $\mathcal{F}_{k}^{Y}$-measurable random variables $U_{k}$ by $\hat{U}_{k}=\hat{v}_{k}\left(\hat{Z}_{k}\right)$ where the functions $\hat{v}_{k}, k=0, \ldots, n$, are defined on $\Gamma_{k}$ by :

$$
\begin{aligned}
\hat{v}_{k}\left(z_{k}^{i}\right) & =\mathbb{E}\left[\hat{\varphi}_{k+1}\left(\hat{Z}_{k+1}\right) \mid \hat{Z}_{k}=z_{k}^{i}\right] \\
& =\sum_{j=1}^{N_{k+1}} \hat{r}_{k+1}^{i j} \hat{\varphi}_{k+1}\left(z_{k+1}^{j}\right), \quad \forall z_{k}^{i} \in \Gamma_{k} .
\end{aligned}
$$




\subsection{The error analysis}

The quality of the approximation described in the previous paragraph is measured as follows. We denote for any subset $D$ in $\mathbb{R}^{l}$ :

$$
\begin{aligned}
B L_{1}(D)= & \{\varphi \text { Borelian from } D \text { into } \mathbb{R}: \\
& \left.\|\varphi\|_{\text {sup }}:=\sup _{x \in D}|\varphi(x)| \leq 1, \quad[\varphi]_{l i p}:=\sup _{x, y \in D, x \neq y} \frac{|\varphi(x)-\varphi(y)|}{|x-y|_{1}} \leq 1\right\} .
\end{aligned}
$$

We make the following assumption.

(H1) There exists a constant $L_{g}$ such that for all $k \geq 1$ :

$$
\sum_{i, j=1}^{m} P_{k}^{i j} \int\left|g_{k}\left(x^{i}, y, x^{j}, y^{\prime}\right)-g_{k}\left(x^{i}, \hat{y}, x^{j}, y^{\prime}\right)\right| d y^{\prime} \leq L_{g}|y-\hat{y}|_{1}, \quad \forall y, \hat{y} \in \mathbb{R}^{d}
$$

Proposition 3.1 Under (H1), we have for any $n$ and $\varphi_{1}, \ldots, \varphi_{n} \in B L_{1}\left(K_{m} \times \mathbb{R}^{d}\right)$ :

$$
\begin{aligned}
& \left|\int \varphi_{1}\left(z_{1}\right) \ldots \varphi_{n}\left(z_{n}\right)\left(R_{1}\left(z_{0}, d z_{1}\right) \ldots R_{n}\left(z_{n-1}, d z_{n}\right)-\hat{r}_{1}\left(z_{0}, d z_{1}\right) \ldots \hat{r}_{n}\left(z_{n-1}, d z_{n}\right)\right)\right| \\
\leq & \sum_{k=1}^{n} \frac{3 \sqrt{m+d}}{2 \bar{L}_{g}-1}\left[\left(2 \bar{L}_{g}\right)^{n-k+1}-1\right]\left\|Z_{k}-\hat{Z}_{k}\right\|_{2}
\end{aligned}
$$

where $\bar{L}_{g}=\max \left(L_{g}, 1\right)$.

We first state a preliminary result on the Lipschitz property of the transition of the Markov chain $\left(Z_{k}\right)_{k}=\left(\Pi_{k}, Y_{k}\right)_{k}$.

Lemma 3.1 Under (H1), we have for all $k \geq 1$ and Borelian function $\varphi$ on $K_{m} \times \mathbb{R}^{d}$ :

$$
\left|R_{k} \varphi(z)-R_{k} \varphi(\hat{z})\right| \leq\left(2[\varphi]_{l i p}+\|\varphi\|_{\text {sup }}\right) \bar{L}_{g}|z-\hat{z}|_{1}, \quad \forall z, \hat{z} \in K_{m} \times \mathbb{R}^{d} .
$$

Proof. Recall from (2.3) that the conditional law $Q_{k}\left(\pi, y, d y^{\prime}\right)$ of $Y_{k}$ given $\left(\Pi_{k-1}, Y_{k-1}\right)=$ $(\pi, y)$ admits a density given by :

$$
y^{\prime} \longmapsto f_{k}\left(\pi, y, y^{\prime}\right)=\sum_{i, j=1}^{m} g_{k}\left(x^{i}, y, x^{j}, y^{\prime}\right) P_{k}^{i j} \pi^{i}
$$

This conditional density satisfies the Lipschitz property : for all $(\pi, y)$ and $(\hat{\pi}, \hat{y}) \in K_{m} \times \mathbb{R}^{d}$,

$$
\begin{aligned}
\int\left|f_{k}\left(\pi, y, y^{\prime}\right)-f_{k}\left(\hat{\pi}, \hat{y}, y^{\prime}\right)\right| d y^{\prime} \leq & \sum_{i, j=1}^{m} P_{k}^{i j} \int\left|g_{k}\left(x^{i}, y, x^{j}, y^{\prime}\right)-g_{k}\left(x^{i}, \hat{y}, x^{j}, y^{\prime}\right)\right| d y^{\prime} \\
& +\sum_{i}^{m}\left|\pi^{i}-\hat{\pi}^{i}\right|
\end{aligned}
$$


where we used the fact that $\sum_{j} P_{k}^{i j} \int g_{k}\left(x^{i}, y, x^{j}, y^{\prime}\right) d y^{\prime}=1$. From (2.4), we then have for any $z=(\pi, y)$ and $\hat{z}=(\hat{\pi}, \hat{y}) \in K_{m} \times \mathbb{R}^{d}$ :

$$
\begin{aligned}
\left|R_{k} \varphi(z)-R_{k} \varphi(\hat{z})\right| \leq & \int\left|\varphi\left(\bar{G}_{k}\left(\pi, y, y^{\prime}\right), y^{\prime}\right)-\varphi\left(\bar{G}_{k}\left(\hat{\pi}, \hat{y}, y^{\prime}\right), y^{\prime}\right)\right| Q_{k}\left(\pi, y, d y^{\prime}\right) \\
& +\left|\int \varphi\left(\bar{G}_{k}\left(\hat{\pi}, \hat{y}, y^{\prime}\right), y^{\prime}\right)\left(Q_{k}\left(\pi, y, d y^{\prime}\right)-Q_{k}\left(\hat{\pi}, \hat{y}, d y^{\prime}\right)\right)\right| \\
\leq & {[\varphi]_{l i p} \int\left|\bar{G}_{k}\left(\pi, y, y^{\prime}\right)-\bar{G}_{k}\left(\hat{\pi}, \hat{y}, y^{\prime}\right)\right|_{1} f_{k}\left(\pi, y, y^{\prime}\right) d y^{\prime} } \\
& \quad+\|\varphi\|_{\text {sup }} \int\left|f_{k}\left(\pi, y, y^{\prime}\right)-f_{k}\left(\hat{\pi}, \hat{y}, y^{\prime}\right)\right| d y^{\prime} \\
\leq & {[\varphi]_{l i p} \int\left|\bar{G}_{k}\left(\pi, y, y^{\prime}\right)-\bar{G}_{k}\left(\hat{\pi}, \hat{y}, y^{\prime}\right)\right|_{1} f_{k}\left(\pi, y, y^{\prime}\right) d y^{\prime} } \\
& \quad+\|\varphi\|_{\text {sup }} \sum_{i, j=1}^{m} P_{k}^{i j} \int\left|g_{k}\left(x^{i}, y, x^{j}, y^{\prime}\right)-g_{k}\left(x^{i}, \hat{y}, x^{j}, y^{\prime}\right)\right| d y^{\prime} \\
& +\|\varphi\|_{\text {sup }} \sum_{i}^{m}\left|\pi^{i}-\hat{\pi}^{i}\right| .
\end{aligned}
$$

Now, from (3.2), we have :

$$
\begin{aligned}
& \int\left|\bar{G}_{k}\left(\pi, y, y^{\prime}\right)-\bar{G}_{k}\left(\hat{\pi}, \hat{y}, y^{\prime}\right)\right|_{1} f_{k}\left(\pi, y, y^{\prime}\right) d y^{\prime} \\
\leq & \sum_{j=1}^{m} \int\left|\bar{G}_{k}^{j}\left(\pi, y, y^{\prime}\right)-\bar{G}_{k}^{j}\left(\hat{\pi}, \hat{y}, y^{\prime}\right)\right| f_{k}\left(\pi, y, y^{\prime}\right) d y^{\prime} \\
= & \sum_{i, j=1}^{m} \int\left|\frac{g_{k}\left(x^{i}, y, x^{j}, y^{\prime}\right) P_{k}^{i j} \pi^{i}}{f_{k}\left(\pi, y, y^{\prime}\right)}-\frac{g_{k}\left(x^{i}, \hat{y}, x^{j}, y^{\prime}\right) P_{k}^{i j} \hat{\pi}^{i}}{f_{k}\left(\hat{\pi}, \hat{y}, y^{\prime}\right)}\right| f_{k}\left(\pi, y, y^{\prime}\right) d y^{\prime} \\
\leq & \sum_{i, j=1}^{m} P_{k}^{i j} \hat{\pi}^{i} \int \frac{\left|g_{k}\left(x^{i}, y, x^{j}, y^{\prime}\right) f_{k}\left(\hat{\pi}, \hat{y}, y^{\prime}\right)-g_{k}\left(x^{i}, \hat{y}, x^{j}, y^{\prime}\right) f_{k}\left(\pi, y, y^{\prime}\right)\right|}{f_{k}\left(\hat{\pi}, \hat{y}, y^{\prime}\right)} d y^{\prime} \\
& +\sum_{i=1}^{m}\left|\pi^{i}-\hat{\pi}^{i}\right| \\
\leq & \sum_{i, j=1}^{m} P_{k}^{i j} \int\left|g_{k}\left(x^{i}, y, x^{j}, y^{\prime}\right)-g_{k}\left(x^{i}, \hat{y}, x^{j}, y^{\prime}\right)\right| d y^{\prime} \\
& +\int\left|f_{k}\left(\pi, y, y^{\prime}\right)-f_{k}\left(\hat{\pi}, \hat{y}, y^{\prime}\right)\right| d y^{\prime}+\sum_{i=1}^{m}\left|\pi^{i}-\hat{\pi}^{i}\right| \\
\leq & 2 \sum_{i, j=1}^{m} P_{k}^{i j} \int\left|g_{k}\left(x^{i}, y, x^{j}, y^{\prime}\right)-g_{k}\left(x^{i}, \hat{y}, x^{j}, y^{\prime}\right)\right| d y^{\prime}+2 \sum_{i=1}^{m}\left|\pi^{i}-\hat{\pi}^{i}\right|,
\end{aligned}
$$

where we used again (3.4). Plugging into (3.5) and using (H1) yield :

$$
\begin{aligned}
& \left|R_{k} \varphi(z)-R_{k} \varphi(\hat{z})\right| \\
\leq & \left(2[\varphi]_{l i p}+\|\varphi\|_{\text {sup }}\right)\left(\sum_{i, j=1}^{m} P_{k}^{i j} \int\left|g_{k}\left(x^{i}, y, x^{j}, y^{\prime}\right)-g_{k}\left(x^{i}, \hat{y}, x^{j}, y^{\prime}\right)\right| d y^{\prime}+\sum_{i=1}^{m}\left|\pi^{i}-\hat{\pi}^{i}\right|\right)
\end{aligned}
$$




$$
\leq\left(2[\varphi]_{l i p}+\|\varphi\|_{\text {sup }}\right)\left(L_{g}|y-\hat{y}|_{1}+|\pi-\hat{\pi}|_{1}\right)
$$

and then the required result.

Remark 3.1 In the case where the law of $Y_{k}$ conditional on $\left(X_{k-1}, Y_{k-1}, X_{k}\right)$ does not depend on $Y_{k-1}$, i.e. its density $g_{k}$ depends only $X_{k-1}, X_{k}$, the condition (H1) is empty, or in other words is trivially satisfied with $L_{g}=0$. Then, inequality (3.6) shows that for all $z=(\pi, y), \hat{z}=(\hat{\pi}, \hat{y}) \in K_{m} \times \mathbb{R}^{d}$,

$$
\left|R_{k} \varphi(z)-R_{k} \varphi(\hat{z})\right| \leq\left(2[\varphi]_{l i p}+\|\varphi\|_{\text {sup }}\right)|\pi-\hat{\pi}|_{1} .
$$

Proof of Proposition 3.1. For $k=1, \ldots, n$, we define the measurable functions on $K_{m} \times \mathbb{R}^{d}$, resp. on $\Gamma_{k}$ :

$$
\begin{aligned}
& v_{k}(z)=\varphi_{k}(z) \int \varphi_{k+1}\left(z_{k+1}\right) \ldots \varphi_{n}\left(z_{n}\right) R_{k+1}\left(z, d z_{k+1}\right) \ldots R_{n}\left(z_{n-1}, d z_{n}\right), \\
& \hat{v}_{k}(z)=\varphi_{k}(z) \int \varphi_{k+1}\left(z_{k+1}\right) \ldots \varphi_{n}\left(z_{n}\right) \hat{r}_{k+1}\left(z, d z_{k+1}\right) \ldots \hat{r}_{n}\left(z_{n-1}, d z_{n}\right),
\end{aligned}
$$

with the convention that for $k=n, v_{n}=\hat{v}_{n}=\varphi_{n}$, we then have the backward induction formulas :

$$
\begin{aligned}
& v_{k}(z)=\varphi_{k}(z) R_{k+1} v_{k+1}(z):=\varphi_{k}(z) \mathbb{E}\left[v_{k+1}\left(Z_{k+1}\right) \mid Z_{k}=z\right] \\
& \hat{v}_{k}(z)=\varphi_{k}(z) \hat{r}_{k+1} \hat{v}_{k+1}(z):=\varphi_{k}(z) \mathbb{E}\left[\hat{v}_{k+1}\left(\hat{Z}_{k+1}\right) \mid \hat{Z}_{k}=z\right]
\end{aligned}
$$

for all $k=1, \ldots, n-1$.

Step 1. We clearly have $\left\|v_{k}\right\|_{\text {sup }} \leq 1$. Moreover, from (3.8) and using Lemma 3.1, we have :

$$
\begin{aligned}
{\left[v_{k}\right]_{l i p} } & \leq\left[\varphi_{k}\right]_{l i p}+\left[R_{k+1} v_{k+1}\right]_{l i p} \\
& \leq 1+\bar{L}_{g}+2 \bar{L}_{g}\left[v_{k+1}\right]_{l i p} .
\end{aligned}
$$

Since $\left[v_{n}\right]_{l i p} \leq 1$, a standard backward induction yields :

$$
\left[v_{k}\right]_{l i p} \leq \frac{\frac{3}{2}\left(2 \bar{L}_{g}\right)^{n-k+1}-1-\bar{L}_{g}}{2 \bar{L}_{g}-1},
$$

for all $k=0, \ldots, n$.

Step 2. From (3.8)-(3.9), we may write :

$$
\begin{aligned}
\left\|v_{k}\left(Z_{k}\right)-\hat{v}_{k}\left(\hat{Z}_{k}\right)\right\|_{1} \leq & \left\|v_{k}\left(Z_{k}\right)-\mathbb{E}\left[v_{k}\left(Z_{k}\right) \mid \hat{Z}_{k}\right]\right\|_{1} \\
& +\left\|\mathbb{E}\left[\left(\varphi_{k}\left(Z_{k}\right)-\varphi_{k}\left(\hat{Z}_{k}\right)\right) R_{k+1} v_{k+1}\left(Z_{k}\right) \mid \hat{Z}_{k}\right]\right\|_{1} \\
& +\left\|\mathbb{E}\left[\varphi\left(\hat{Z}_{k}\right)\left(R_{k+1} v_{k+1}\left(Z_{k}\right)-\hat{r}_{k+1} \hat{v}_{k+1}\left(\hat{Z}_{k}\right)\right) \mid \hat{Z}_{k}\right]\right\|_{1} \\
= & I_{1}+I_{2}+I_{3} .
\end{aligned}
$$


By the law of iterated conditional expectation, we have :

$$
\begin{aligned}
I_{1} & \leq\left\|v_{k}\left(Z_{k}\right)-v_{k}\left(\hat{Z}_{k}\right)\right\|_{1}+\left\|\mathbb{E}\left[v_{k}\left(\hat{Z}_{k}\right) \mid \hat{Z}_{k}\right]-\mathbb{E}\left[v_{k}\left(Z_{k}\right) \mid \hat{Z}_{k}\right]\right\|_{1} \\
& \leq 2\left\|v_{k}\left(Z_{k}\right)-v_{k}\left(\hat{Z}_{k}\right)\right\|_{1} \leq 2\left[v_{k}\right]_{l i p}\left\|Z_{k}-\hat{Z}_{k}\right\|_{1} .
\end{aligned}
$$

Since conditional expectation (here with respect to $\hat{Z}_{k}$ ) is a $L^{1}$-contraction, and $v_{k+1}$ is bounded by 1 , we have :

$$
I_{2} \leq\left\|\varphi_{k}\left(Z_{k}\right)-\varphi_{k}\left(\hat{Z}_{k}\right)\right\|_{1} \leq\left\|Z_{k}-\hat{Z}_{k}\right\|_{1}
$$

recalling that $\varphi_{k}$ is in $B L_{1}\left(K_{m} \times \mathbb{R}^{d}\right)$. Since $\hat{Z}_{k}$ is $\sigma\left(Z_{k}\right)$-measurable, and recalling also that $\varphi_{k}$ is bounded by 1 , we have :

$$
I_{3} \leq\left\|v_{k+1}\left(Z_{k+1}\right)-\hat{v}_{k+1}\left(\hat{Z}_{k+1}\right)\right\|_{1} .
$$

Plugging these estimates of $I_{1}, I_{2}$ and $I_{3}$ into (3.11), we get

$$
\left\|v_{k}\left(Z_{k}\right)-\hat{v}_{k}\left(\hat{Z}_{k}\right)\right\|_{1} \leq\left(1+2\left[v_{k}\right]_{l i p}\right)\left\|Z_{k}-\hat{Z}_{k}\right\|_{1}+\left\|v_{k+1}\left(Z_{k+1}\right)-\hat{v}_{k+1}\left(\hat{Z}_{k+1}\right)\right\|_{1} .
$$

Since $\left\|v_{n}\left(Z_{n}\right)-\hat{v}_{n}\left(\hat{Z}_{n}\right)\right\|_{1} \leq\left\|Z_{n}-\hat{Z}_{n}\right\|_{1}$, a direct backward induction yields :

$$
\left\|v_{k}\left(Z_{k}\right)-\hat{v}_{k}\left(\hat{Z}_{k}\right)\right\|_{1} \leq \sum_{j=k}^{n}\left(1+2\left[v_{j}\right]_{l i p}\right)\left\|Z_{j}-\hat{Z}_{j}\right\|_{1} .
$$

The required result is proved by taking $k=0$, substituting the estimate (3.10) and using Cauchy-Schwarz inequality : $\left\|Z_{j}-\hat{Z}_{j}\right\|_{1} \leq \sqrt{m+d}\left\|Z_{j}-\hat{Z}_{j}\right\|_{2}$.

\subsection{Optimal quantization and rate of convergence}

The estimation error (3.3) in Proposition 3.1 shows that to obtain the best approximation of the sequence of probability transitions $\left(R_{k}\right)$ of the Markov chain $\left(Z_{k}\right)_{k}=\left(\Pi_{k}, Y_{k}\right)_{k}$ by this quantization approach, one has to minimize at each time $k \geq 1$ the $L^{2}$ quantization error $\left\|Z_{k}-\hat{Z}_{k}\right\|_{2}$. By identifying a grid $\Gamma_{k}=\left\{z^{1}, \ldots, z^{N_{k}}\right\}$ of size $\left|\Gamma_{k}\right|=N_{k}$ points in $K_{m} \times \mathbb{R}^{d}$, with the $N_{k^{-}}$tuple $\left(z^{1}, \ldots, z^{N_{k}}\right) \in\left(K_{m} \times \mathbb{R}^{d}\right)^{N_{k}}$, the objective is then to minimize the symmetric function :

$$
\begin{aligned}
D_{N_{k}}^{Z_{k}}\left(z^{1}, \ldots, z^{N_{k}}\right) & =\left\|Z_{k}-\operatorname{Proj}_{\Gamma_{k}}\left(Z_{k}\right)\right\|_{2}^{2} \\
& =\mathbb{E}\left[\min _{1 \leq i \leq N_{k}}\left|Z_{k}-z^{i}\right|_{2}^{2}\right], \forall \Gamma_{k}=\left(z^{1}, \ldots, z^{N_{k}}\right) \in\left(K_{m} \times \mathbb{R}^{d}\right)^{N_{(}}
\end{aligned}
$$

which is the square of the $L^{2}$ quantization error and is usually called distorsion. The optimal quantization consists, for each $k \geq 1$ and given a number of points $N_{k}$, to find a grid $\hat{\Gamma}_{k}$ of size $N_{k}$ that reaches the minimum of the distorsion function $D_{N_{k}}^{Z_{k}}$. This question has been tackled for a long time as part of quantization for information theory and signal processing, and more recently in probability for both numerical and theoretical purpose (see [7] or [11]). 
We recall these results and apply them in our context in the next paragraph. Now, we focus on the behaviour of the minimum of this function, i.e. the minimal $L^{2}$ quantization error, when the number of points $N_{k}$ goes to infinity. For this, we first recall the so-called Zador theorem, see e.g. [7].

Theorem 3.1 Let $X$ be a $\mathbb{R}^{l}$-valued random variable with distribution $\mathbb{P}_{X}$, s.t. $\mathbb{E}|X|_{2}^{2+\delta}<$ $\infty$ for some $\delta>0$. Then

$$
\lim _{N}\left(N^{\frac{2}{l}} \min _{|\Gamma| \leq N}\left\|X-\operatorname{Proj}_{\Gamma}(X)\right\|_{2}^{2}\right)=J_{2, l}\left(\int_{\mathbb{R}^{l}}|f|^{\frac{l}{l+2}}(x) d x\right)^{\frac{l+2}{l}}
$$

where $\mathbb{P}_{X}(d x)=f(x) \lambda_{l}(d x)+\nu(d x)$ is the Lebesgue decomposition of $\mathbb{P}_{X}$ with respect to the Lebesgue measure $\lambda_{l}$ on $\mathbb{R}^{l}$. The constant $J_{2, l}$ corresponds to the case where $X$ is the uniform distribution on $[0,1]^{l}$.

Remark 3.2 In dimension $l=1$ and 2 , the values of $J_{2, l}$ are known : $J_{2,1}=1 / 6$ and $J_{2,2}$ $=\frac{5}{18 \sqrt{3}}$. In higher dimension, the true value of $J_{2, l}$ is unknown but we have an equivalent $J_{2, l} \sim \frac{l}{2 \pi e}$ as $l$ goes to infinity.

Here, we cannot apply directly this theorem to $Z_{k}$ since the distribution $\mathbb{P}_{Z_{k}}$ of $\left(\Pi_{k}, Y_{k}\right)$ is not known in general, and in particular its decomposition with respect to the Lebesgue measure. However, one can prove the following error bound for the minimal distorsion error of $Z_{k}$.

Proposition 3.2 For $k \geq 1$, assume that there exists some $\varepsilon>0$ s.t. :

$$
\int\left|y_{k}\right|_{2}^{2+\varepsilon} \prod_{l=1}^{k} g_{l}\left(x_{l-1}, y_{l-1}, x_{l}, y_{l}\right) d y_{l}<\infty, \quad \forall x_{0}, \ldots, x_{k} \in E .
$$

Then, we have :

$$
\limsup _{N_{k} \rightarrow \infty} N_{k}^{\frac{2}{m-1+d}} \min _{\left|\Gamma_{k}\right| \leq N_{k}}\left\|Z_{k}-\operatorname{Proj}_{\Gamma_{k}}\left(Z_{k}\right)\right\|_{2}^{2} \leq C\left(m, d, f_{k}\right),
$$

where

$$
C\left(m, d, f_{k}\right)=\frac{m(d+m-1)}{(m d)^{\frac{d}{d+m-1}}}\left(J_{2, d}\right)^{\frac{d}{d+m-1}}\left(\int_{\mathbb{R}^{d}}\left|f_{k}\right|^{\frac{d}{d+2}}(y) d y\right)^{\frac{d+2}{d+m-1}},
$$

and $f_{k}$ is the marginal density of $Y_{k}$ given by :

$f_{k}(y)=\int g_{k}\left(x_{k-1}, y_{k-1}, x_{k}, y\right) P_{k}\left(x_{k-1}, d x_{k}\right) \prod_{l=1}^{k-1} g_{l}\left(x_{l-1}, y_{l-1}, x_{l}, y_{l}\right) P_{l}\left(x_{l-1}, d x_{l}\right) d y_{l} \mu\left(d x_{0}\right)$. 
Remark 3.3 In the case where the law of $Y_{k}$ conditional on $\left(X_{k-1}, Y_{k-1}, X_{k}\right)$ does not depend on $Y_{k-1}$, i.e. the function $g_{k}$ does not depend on $Y_{k-1}$, the expression $f_{k}$ of the marginal density of $Y_{k}$ simplifies into :

$$
f_{k}(y)=\mathbb{E}\left[g_{k}\left(X_{k-1}, X_{k}, y\right)\right], \quad y \in \mathbb{R}^{d}
$$

and condition (3.14) is written as :

$$
\int\left|y_{k}\right|_{2}^{2+\delta} g_{k}\left(x_{k-1}, x_{k}, y_{k}\right) d y_{k}<\infty, \quad \forall x_{k-1}, x_{k} \in E
$$

for some $\delta>0$.

Proof of Proposition 3.2. For any grids

$$
\begin{aligned}
& \Gamma^{\Pi}=\{\pi(1), \ldots, \pi(M)\} \quad \text { of size }\left|\Gamma^{\Pi}\right|=M \text { points in } K_{m}, \\
& \Gamma^{Y}=\left\{y^{1}, \ldots, y^{L}\right\} \quad \text { of size }\left|\Gamma^{Y}\right|=L \text { points in } \mathbb{R}^{d},
\end{aligned}
$$

we denote

$$
\Gamma^{\Pi} \otimes \Gamma^{Y}=\left\{\left(\pi(i), y^{j}\right): 1 \leq i \leq M, 1 \leq j \leq L\right\} \quad \text { of size } M L \text { points in } K_{m} \times \mathbb{R}^{d} .
$$

We then have by definition of the norm $\|\cdot\|_{2}$ and of the projection :

$$
\left\|Z_{k}-\operatorname{Proj}_{\Gamma^{\Pi} \otimes \Gamma^{Y}}\left(Z_{k}\right)\right\|_{2}^{2}=\left\|\Pi_{k}-\operatorname{Proj}_{\Gamma^{\Pi}}\left(\Pi_{k}\right)\right\|_{2}^{2}+\left\|Y_{k}-\operatorname{Proj}_{\Gamma^{Y}}\left(Y_{k}\right)\right\|_{2}^{2},
$$

and so

$$
\begin{aligned}
& \min _{\left|\Gamma_{k}\right| \leq N_{k}}\left\|Z_{k}-\operatorname{Proj}_{\Gamma_{k}}\left(Z_{k}\right)\right\|_{2}^{2} \\
\leq & \min _{M, L: M L \leq N_{k}}\left(\min _{\left|\Gamma^{\Pi}\right| \leq M}\left\|\Pi_{k}-\operatorname{Proj}_{\Gamma^{\Pi}}\left(\Pi_{k}\right)\right\|_{2}^{2}+\min _{\left|\Gamma^{Y}\right| \leq L}\left\|Y_{k}-\operatorname{Proj}_{\Gamma^{Y}}\left(Y_{k}\right)\right\|_{2}^{2}\right)
\end{aligned}
$$

For $M_{0}$ in $\mathbb{N} \backslash\{0\}$, consider the grid $\Gamma^{\Pi}$ of size $M=M_{0}^{m-1}$ points in $K_{m}$ :

$$
\Gamma^{\Pi}=\left\{\left(\frac{i_{1}}{M_{0}}, \ldots, \frac{i_{m-1}}{M_{0}}, 1-\sum_{l=1}^{m-1} \frac{i_{l}}{M_{0}}\right): i_{1}, \ldots, i_{m-1}=1, \ldots, M_{0}, \sum_{l=1}^{m-1} i_{l} \leq M_{0}\right\}
$$

Denoting for all $a \in \mathbb{R},[a]$ the smallest integer smaller than $a$, we have for all $\pi=\left(\pi^{i}\right)_{1 \leq i \leq m}$ $\in K_{m}$ :

$$
\begin{aligned}
\left|\pi-\operatorname{Proj}_{\Gamma}(\pi)\right|_{2}^{2} & =\min _{\substack{i_{1}, \ldots, i_{m-1}=1, \ldots, M_{0} \\
i_{1}+\ldots+i_{m-1} \leq M_{0}}} \sum_{l=1}^{m-1}\left|\pi^{l}-\frac{i_{l}}{M_{0}}\right|^{2}+\left|\sum_{l=1}^{m-1}\left(\pi^{l}-\frac{i_{l}}{M_{0}}\right)\right|^{2} \\
& \leq m \min _{\substack{i_{1}, \ldots, i_{m-1}=1, \ldots, M_{0} \\
i_{1}+\ldots+i_{m-1} \leq M_{0}}} \sum_{l=1}^{m-1}\left|\pi^{l}-\frac{i_{l}}{M_{0}}\right|^{2} \\
& \leq m \sum_{l=1}^{m-1}\left|\pi^{l}-\frac{\left[\pi^{l} M_{0}\right]}{M_{0}}\right|^{2} \\
& \leq \frac{m(m-1)}{M_{0}^{2}} .
\end{aligned}
$$


This shows that:

$$
\min _{\left|\Gamma^{\Pi}\right| \leq M}\left\|\Pi_{k}-\operatorname{Proj}_{\Gamma^{\Pi}}\left(\Pi_{k}\right)\right\|_{2}^{2} \leq \frac{m(m-1)}{M^{\frac{2}{m-1}}} .
$$

On the other hand, notice that condition (3.14) ensures that $\mathbb{E}\left|Y_{k}\right|_{2}^{2+\varepsilon}<\infty$. Thus, from Theorem 3.1 applied to $Y_{k}$, we have for all $\delta>0$ and $L$ large enough,

$$
\min _{\left|\Gamma^{Y}\right| \leq L}\left\|Y_{k}-\operatorname{Proj}_{\Gamma^{Y}}\left(Y_{k}\right)\right\|_{2}^{2} \leq \frac{\left(J_{2, d}\left\|f_{k}\right\|_{\frac{d}{d+2}}+\delta\right)}{L^{\frac{2}{d}}},
$$

where we set :

$$
\left\|f_{k}\right\|_{\frac{d}{d+2}}=\left(\int_{\mathbb{R}^{d}}\left|f_{k}\right|^{\frac{d}{d+2}}(y) d y\right)^{\frac{d+2}{d}} .
$$

Substituting (3.17) and (3.18) into (3.16) yields :

$$
\begin{aligned}
& \min _{\left|\Gamma_{k}\right| \leq N_{k}}\left\|Z_{k}-\operatorname{Proj}_{\Gamma_{k}}\left(Z_{k}\right)\right\|_{2}^{2} \\
\leq & \min _{M, L: M L \leq N_{k}}\left[\frac{m(m-1)}{M^{\frac{2}{m-1}}}+\frac{\left(J_{2, d}\left\|f_{k}\right\|_{\frac{d}{d+2}}+\delta\right)}{L^{\frac{2}{d}}}\right] .
\end{aligned}
$$

We conclude with the elementary result that for all $a, b>0$ :

$$
\min _{M, L: M L \leq N}\left[\frac{a}{M^{\frac{2}{l}}}+\frac{b}{L^{\frac{2}{d}}}\right]=(d+l)\left(\frac{a}{l}\right)^{\frac{l}{d+l}}\left(\frac{b}{d}\right)^{\frac{d}{d+l}} \frac{1}{N^{\frac{2}{d+l}}} .
$$

\subsection{Practical implementation of the optimal approximating filter process}

We now come back to the numerical implementation of an algorithm that computes for each $k$ :

- an optimal grid $\hat{\Gamma}_{k}$ which minimizes the distorsion :

$$
D_{N_{k}}^{Z_{k}}\left(\Gamma_{k}\right)=\left\|Z_{k}-\operatorname{Proj}_{\Gamma_{k}}\left(Z_{k}\right)\right\|_{2}^{2}
$$

as well as an estimation of this error,

- the weights of the Voronoi tesselations :

$$
\begin{aligned}
\hat{p}_{k}^{i} & =\mathbb{P}\left[Z_{k} \in C_{i}\left(\hat{\Gamma}_{k}\right)\right], \quad i=1, \ldots, N_{k}, \\
\hat{\beta}_{k+1}^{i j} & =\mathbb{P}\left[Z_{k+1} \in C_{j}\left(\hat{\Gamma}_{k+1}\right), Z_{k} \in C_{i}\left(\hat{\Gamma}_{k}\right)\right], \quad i=1, \ldots, N_{k}, j=1, \ldots, N_{k+1},
\end{aligned}
$$

and so the probability transition matrix $\hat{r}_{k+1}^{i j}=\hat{\beta}_{k+1}^{i j} / \hat{p}_{k}^{i}$. 
This program is based on the following key property of the distorsion : The function $D_{N_{k}}^{Z_{k}}$ is continuously differentiable at any $N_{k^{-}}$tuple $\Gamma_{k}=\left(z^{1}, \ldots, z^{N_{k}}\right) \in\left(K_{m} \times \mathbb{R}^{d}\right)^{N_{k}}$ having pairwise distinct components and its gradient is obtained by formal differentiation inside the expectation operator in (3.12) (see [11]) :

$$
\nabla D_{N_{k}}^{Z_{k}}\left(\Gamma_{k}\right)=2 \mathbb{E}\left[H\left(\Gamma_{k}, Z_{k}\right)\right]
$$

where the $\left(K_{m} \times \mathbb{R}^{d}\right)^{N_{k}}$-vector valued function $H$ is given by :

$H\left(\Gamma_{k}, z\right)=\left(\left(z^{i}-z\right) 1_{z \in C_{i}\left(\Gamma_{k}\right)}\right)_{1 \leq i \leq N_{k}}, \quad \Gamma_{k}=\left(z^{1}, \ldots, z^{N_{k}}\right) \in\left(K_{m} \times \mathbb{R}^{d}\right)^{N_{k}}, z \in K_{m} \times \mathbb{R}^{d}$.

This above integral representation for $\nabla D_{N_{k}}^{Z_{k}}$ suggests to implement a stochastic gradient descent, whenever one is able to simulate easily independent copies of $Z_{k}$. We will come back below on the simulation of $Z_{k}$. The stochastic gradient procedure is recursively defined by

$$
\Gamma_{k}^{s+1}=\Gamma_{k}^{s}-\delta_{s+1} H\left(\Gamma_{k}^{s}, \xi_{k}^{s+1}\right)
$$

where the initial grid $\Gamma_{k}^{0}$ has $N_{k}$ pairwise distinct components, $\left(\xi_{k}^{s}\right)_{s \geq 1}$ is an i.i.d. sequence of $\mathbb{P}_{z_{k}}$-distributed random vectors, and $\left(\delta_{s}\right)_{s \geq 1}$ is a sequence of step parameters satisfying the usual conditions :

$$
\sum_{s} \delta_{s}=\infty \text { and } \quad \sum_{s} \delta_{s}^{2}<\infty
$$

In an abstract framework (see e.g. [6] or [9]), under some appropriate assumptions, a stochastic gradient descent associated to the integral representation of a so-called potential function $\left(D_{N_{k}}^{Z_{k}}\right.$ in our problem) converges a.s., when $s$ goes to infinity, toward a local minimum $\hat{\Gamma}_{k}$ of this potential function :

$$
\nabla D_{N_{k}}^{Z_{k}}\left(\hat{\Gamma}_{k}\right)=0
$$

Although these assumptions are not fulfilled by $D_{N_{k}}^{Z_{k}}$, the encountered theoretical problems can be partially overcome (see [11]) Practical implementation does provide satisfactory results (a commonly encountered situation with gradient descents). Moreover, computation of the weights of the tesselations and of the distorsion can be implemented as by-product of the procedure. We now describe this algorithm, known as the Competitive Learning Vector Quantization algorithm.

Simulation of the Markov chain $\left(Z_{k}\right)_{k}$ :

We notice that from (2.4), we are able to simulate the probability transition $R_{k}$ of the $\left(\mathbb{P}, \mathcal{F}_{k}^{Y}\right)$ Markov chain $\left(Z_{k}\right)_{k}=\left(\Pi_{k}, Y_{k}\right)_{k}$. For $k=0$, recall that $Z_{0}$ is a known deterministic vector equal to $z_{0}=\left(\mu, y_{0}\right)$. For $k \geq 1$, starting from $\left(\Pi_{k-1}, Y_{k-1}\right)$,

- we simulate $X_{k-1}$ with probability law $\Pi_{k-1}$, and then $X_{k}$ according to the probability transition $P_{k}$.

- we simulate $Y_{k}$ according to the probability transition $q_{k}\left(X_{k-1}, Y_{k-1}, X_{k}, d y^{\prime}\right)$. 
- we compute $\Pi_{k}$ by the formula (3.1) :

$$
\Pi_{k}=\frac{G P\left(Y_{k-1}, Y_{k}\right)^{\top} \Pi_{k-1}}{\left|G P\left(Y_{k-1}, Y_{k}\right)^{\top} \Pi_{k-1}\right|_{1}} .
$$

Subsequently, we stock $S$ independent copies of the Markov chain $\left(Z_{0}, \ldots, Z_{n}\right)$, that we denote $\xi^{s}=\left(\xi_{0}^{s}, \ldots, \xi_{n}^{s}\right), s=1, \ldots, S$. The algorithm reads as follows :

\section{Initialisation phase :}

- Initialize the $n$ grids $\Gamma_{k}^{0}=\left(z_{k}^{0,1}, \ldots, z_{k}^{0, N_{k}}\right) \in\left(K_{m} \times \mathbb{R}^{d}\right)^{N_{k}}$ for $k=0, \ldots, n$, with $\Gamma_{0}^{0}$ $=z_{0}$ reduced to $N_{0}=1$ point for $k=0$.

- Initialize the weights vectors : $p_{k}^{0, i}=1 / N_{k}, \beta_{k+1}^{0, i j}=0, i=1, \ldots, N_{k}, j=1, \ldots, N_{k+1}$, and the distorsion $D_{N_{k}}^{0}=0$, for $k=0, \ldots, n$.

Updating $s \rightarrow s+1:$ At step $s$, the $n$ grids $\Gamma_{k}^{s}=\left(z_{k}^{s, 1}, \ldots, z_{k}^{s, N_{k}}\right)$, the weights vectors $p_{k}^{s, i}, \beta_{k+1}^{s, i j}, i=1, \ldots, N_{k}, j=1, \ldots, N_{k+1}$, the distorsion $D_{N_{k}}^{s}$ have been obtained and we use the sample $\xi^{s+1}$ of $\left(Z_{0}, \ldots, Z_{n}\right)$ to update them as follows : for all $k=0, \ldots, n$,

- Competitive phase : select $i_{k}(s+1) \in\left\{1, \ldots, N_{k}\right\}$ such that

$$
\xi^{s+1} \in C_{i_{k}(s+1)}\left(\Gamma_{k}^{s}\right), \quad \text { i.e. } \quad i_{k}(s+1) \in \operatorname{argmin}_{1 \leq i \leq N_{k}}\left|z_{k}^{s, i}-\xi^{s+1}\right|_{2} .
$$

- Learning phase :

$\star$ Updating of the grid :

$$
z_{k}^{s+1, i}=z_{k}^{s, i}-\delta_{s+1} 1_{i=i_{k}(s+1)}\left(z_{k}^{s, i}-\xi^{s+1}\right), \quad i=1, \ldots, N_{k}
$$

$\star$ Updating of the weights vectors and of the probability transition

$$
\begin{aligned}
p_{k}^{s+1, i} & =p_{k}^{s, i}-\delta_{s+1}\left(p_{k}^{s, i}-1_{i=i_{k}(s+1)}\right), \\
\beta_{k+1}^{s+1, i j} & =\beta_{k+1}^{s, i j}-\delta_{s+1}\left(\beta_{k+1}^{s, i j}-1_{i=i_{k}(s+1), j=i_{k+1}(s+1)}\right), \\
r_{k+1}^{s+1, i j} & =\frac{\beta_{k+1}^{s+1, i j}}{p_{k}^{s+1, i}},
\end{aligned}
$$

for all $i=1, \ldots, N_{k}, j=1, \ldots, N_{k+1}$.

$\star$ Updating of the distorsion

$$
D_{N_{k}}^{s+1}=D_{N_{k}}^{s}-\delta_{s+1}\left(D_{N_{k}}^{s}-\left|z_{k}^{s, i_{k}(s+1)}-\xi^{s+1}\right|_{2}^{2}\right)
$$

It is shown in [11] that on the event $\left\{\Gamma_{k}^{s} \rightarrow \hat{\Gamma}_{k}\right\}$, set of trajectories of $\left(\Gamma_{k}^{s}\right)_{s}$ that converge to $\hat{\Gamma}_{k}$ local minimum of the distorsion, we have :

$$
\begin{aligned}
& p_{k}^{s, i} \longrightarrow \hat{p}_{k}^{i}=\mathbb{P}\left[Z_{k} \in C_{i}\left(\hat{\Gamma}_{k}\right)\right], \text { a.s. } \\
& \beta_{k+1}^{s, i j} \longrightarrow \hat{\beta}_{k+1}^{i j}=\mathbb{P}\left[Z_{k+1} \in C_{j}\left(\hat{\Gamma}_{k+1}\right), Z_{k} \in C_{i}\left(\hat{\Gamma}_{k}\right], \quad\right. \text { a.s. } \\
& D_{N_{k}}^{s} \longrightarrow D_{N_{k}}^{Z_{k}}\left(\hat{\Gamma}_{k}\right), \text { a.s. }
\end{aligned}
$$

for all $k=0, \ldots, n, i=1, \ldots, N_{k}, j=1, \ldots, N_{k+1}$, as $s$ goes to infinity. 


\section{Application : optimal stopping under partial observation}

We consider the framework of Section 3. We denote by $\mathcal{T}_{n}^{Y}$ the set of stopping times adapted with respect to the observation filtration $\left(\mathcal{F}_{k}^{Y}\right)$ and valued in $\{0, \ldots, n\}$. Given two measurable functions $f$ and $h$ on $E \times \mathbb{R}^{d}$, we consider the following optimal stopping problem under partial observation :

$$
u_{0}=\sup _{\tau \in \mathcal{T}_{n}^{Y}} \mathbb{E}\left[\sum_{k=0}^{\tau} f\left(X_{k}, Y_{k}\right)+h\left(X_{\tau}, Y_{\tau}\right)\right] .
$$

We shall transform this problem into an optimal stopping problem under complete observation. We denote

$$
J(\tau)=\mathbb{E}\left[\sum_{k=0}^{\tau} f\left(X_{k}, Y_{k}\right)+h\left(X_{\tau}, Y_{\tau}\right)\right], \quad \tau \in \mathcal{T}_{n}^{Y},
$$

the expected gain function associated to (4.1). We shall also introduce the functions :

$$
\begin{aligned}
& \hat{f}(\pi, y)=\sum_{i=1}^{m} f\left(x^{i}, y\right) \pi^{i}, \quad \forall \pi=\left(\pi^{i}\right)_{i} \in K_{m}, \forall y \in \mathbb{R}^{d}, \\
& \hat{h}(\pi, y)=\sum_{i=1}^{m} h\left(x^{i}, y\right) \pi^{i}, \quad \forall \pi=\left(\pi^{i}\right)_{i} \in K_{m}, \forall y \in \mathbb{R}^{d} .
\end{aligned}
$$

Then, by using the law of iterated conditional expectations and the definition of the filter $\left(\Pi_{k}\right)$, we have for all $\tau \in \mathcal{T}_{n}^{Y}$ :

$$
\begin{aligned}
J(\tau) & =\mathbb{E}\left[\sum_{j=0}^{n} 1_{\tau=j}\left(\sum_{k=0}^{j} f\left(X_{k}, Y_{k}\right)+h\left(X_{j}, Y_{j}\right)\right)\right] \\
& =\mathbb{E}\left[\sum_{j=0}^{n} 1_{\tau=j}\left(\sum_{k=0}^{j} \mathbb{E}\left[f\left(X_{k}, Y_{k}\right) \mid \mathcal{F}_{k}^{Y}\right]+\mathbb{E}\left[h\left(X_{j}, Y_{j}\right) \mid \mathcal{F}_{j}^{Y}\right]\right)\right] \\
& =\mathbb{E}\left[\sum_{j=0}^{n} 1_{\tau=j}\left(\sum_{k=0}^{j} \hat{f}\left(\Pi_{k}, Y_{k}\right)+\hat{h}\left(\Pi_{j}, Y_{j}\right)\right)\right] \\
& =\mathbb{E}\left[\sum_{k=0}^{\tau} \hat{f}\left(Z_{k}\right)+\hat{h}\left(Z_{\tau}\right)\right]
\end{aligned}
$$

Therefore, problem (4.1) may be rewritten as :

$$
u_{0}=\sup _{\tau \in \mathcal{T}_{n}^{Y}} \mathbb{E}\left[\sum_{k=0}^{\tau} \hat{f}\left(Z_{k}\right)+\hat{h}\left(Z_{\tau}\right)\right]
$$

Since the process $\left(Z_{k}\right)_{k}=\left(\Pi_{k}, Y_{k}\right)_{k}$ is a $\left(\mathbb{P},\left(\mathcal{F}_{k}^{Y}\right)\right)$ Markov chain, problem (4.2) is then an optimal stopping problem under complete observation. 
By the dynamic programming principle, we have $u_{0}=v_{0}\left(z_{0}\right)$, where the sequence of measurable functions $v_{k}: K_{m} \times \mathbb{R}^{d} \mapsto \mathbb{R}, k=0, \ldots, n$, is given in recursive form by the backward formula :

$$
\begin{gathered}
v_{n}(z)=\hat{h}(z), \quad \forall z \in K_{m} \times \mathbb{R}^{d} \\
v_{k}(z)=\max \left\{\hat{h}(z) ; \mathbb{E}\left[\hat{f}\left(Z_{k+1}\right)+v_{k+1}\left(Z_{k+1}\right) \mid Z_{k}=z\right]\right\}, \\
\forall z \in K_{m} \times \mathbb{R}^{d}, \quad k=0, \ldots, n-1 .
\end{gathered}
$$

Applying the quantization approach described in Section 3 and setting :

$$
\hat{Z}_{k}=\operatorname{Proj}_{\Gamma_{k}}\left(Z_{k}\right), \quad \Gamma_{k}=\left(z_{k}^{1}, \ldots, z_{k}^{N_{k}}\right) \in\left(K_{m} \times \mathbb{R}^{d}\right)^{N_{k}}, k=0, \ldots, n,
$$

we then approximate, following [1], the sequence of functions $\left(v_{k}\right)$ by the sequence of functions $\hat{v}_{k}: \Gamma_{k} \rightarrow \mathbb{R}, k=0, \ldots, n$ defined by :

$$
\begin{gathered}
\hat{v}_{n}(z)=\hat{h}(z), \quad \forall z \in \Gamma_{n} \\
\hat{v}_{k}(z)=\max \left\{\hat{h}(z) ; \mathbb{E}\left[\hat{f}\left(\hat{Z}_{k+1}\right)+\hat{v}_{k+1}\left(\hat{Z}_{k+1}\right) \mid \hat{Z}_{k}=z\right]\right\}, \\
\forall z \in \Gamma_{k}, \quad k=0, \ldots, n-1 .
\end{gathered}
$$

From an algorithmic viewpoint, this reads as follows :

$$
\begin{aligned}
\hat{v}_{n}\left(z_{n}^{i}\right)=\hat{h}\left(z_{n}^{i}\right), \quad i=1, \ldots, N_{n} \\
\hat{v}_{k}\left(z_{k}^{i}\right)=\max \left\{\hat{h}\left(z_{k}^{i}\right) ; \sum_{j=1}^{N_{k+1}} \hat{r}_{k+1}^{i j}\left(\hat{f}\left(z_{k+1}^{j}\right)+\hat{v}_{k+1}\left(z_{k+1}^{j}\right)\right)\right\}, \\
i=1, \ldots, N_{k}, \quad k=0, \ldots, n-1 .
\end{aligned}
$$

The optimal grids $\Gamma_{k}$ and the associated probability transition matrix $\left(\hat{r}_{k+1}^{i j}\right)$ are estimated following the procedure described in Section 3.

The induced approximation error is provided in the following theorem.

Theorem 4.1 Assume that $f$ and $h$ are bounded and Lipschitz, uniformly in $x \in E$, i.e.

$$
\|f\|_{\text {sup }}:=\sup _{x \in E, y \in \mathbb{R}^{d}}|f(x, y)|<\infty \quad \text { and } \quad[f]_{l i p}:=\sup _{x \in E, y, \hat{y} \in \mathbb{R}^{d}, y \neq \hat{y}} \frac{|f(x, y)-f(x, \hat{y})|}{|y-\hat{y}|_{1}}<\infty .
$$

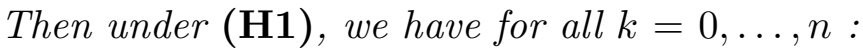

$$
\begin{aligned}
& \left\|v_{k}\left(Z_{k}\right)-\hat{v}_{k}\left(\hat{Z}_{k}\right)\right\|_{1} \\
\leq & \sqrt{m+d}(\bar{f}+\bar{h}) \sum_{j=k}^{n}\left[(7+2(n-j)) \bar{L}_{g}+(n-j+2) \frac{\left(2 \bar{L}_{g}\right)^{n-j+1}}{2 \bar{L}_{g}-1}\right]\left\|Z_{j}-\hat{Z}_{j}\right\|_{2},
\end{aligned}
$$

where $\bar{f}=\max \left(\|f\|_{\text {sup }},[f]_{\text {lip }}\right), \bar{h}=\max \left(\|h\|_{\text {sup }},[h]_{\text {lip }}\right)$. 
Remark 4.1 In view of Proposition 3.2, the estimation (4.5) provides a rate of convergence for the approximation of $v_{0}\left(Z_{0}\right)$ of order

$$
\frac{n\left(2 \bar{L}_{g}\right)^{n}}{N^{\frac{1}{m-1+d}}},
$$

when $N_{k}=N$ is the number of points at each grid $\Gamma_{k}$ used for the optimal quantization of $Z_{k}, k=0, \ldots, n$. The term $\left(2 \bar{L}_{g}\right)^{n}$ is important when $n$ is large, but this is consistent with the rate of convergence obtained in approximation of nonlinear filtering by quantization, see [12] or by Monte-Carlo particle methods, see [4].

Lemma 4.1 Under the assumptions of Theorem 4.1, the functions $v_{k}, k=0, \ldots, n$, defined in (4.4), are bounded and Lipschitz with :

$$
\begin{gathered}
\left\|v_{k}\right\|_{\text {sup }} \leq(n-k+1)(\bar{f}+\bar{h}), \\
{\left[v_{k}\right]_{l i p} \leq \frac{n-k+3}{2} \frac{(\bar{f}+\bar{h})}{2 \bar{L}_{g}-1}\left(2 \bar{L}_{g}\right)^{n-k+1} .}
\end{gathered}
$$

Proof. From (4.4), we have $\left\|v_{k}\right\|_{\text {sup }} \leq\|\hat{h}\|_{\text {sup }}+\|\hat{f}\|_{\text {sup }}+\left\|v_{k+1}\right\|_{\text {sup }}$. Since $\left\|v_{n}\right\|_{\text {sup }}$ $=\|\hat{h}\|_{\text {sup }},\|\hat{f}\|_{\text {sup }} \leq\|f\|_{\text {sup }},\|\hat{h}\|_{\text {sup }} \leq\|h\|_{\text {sup }}$, we obtain immediately the inequality (4.6) by induction.

On the other hand, relation (4.4) and Lemma 3.1 also show

$$
\begin{aligned}
{\left[v_{k}\right]_{l i p} } & \leq[\hat{h}]_{l i p}+\left[R_{k+1}\left(\hat{f}+v_{k+1}\right)\right]_{l i p} \\
& \leq[\hat{h}]_{l i p}+\bar{L}_{g}\left(\|\hat{f}\|_{\text {sup }}+2[\hat{f}]_{l i p}+\left\|v_{k+1}\right\|_{\text {sup }}+2\left[v_{k+1}\right]_{l i p}\right) .
\end{aligned}
$$

By definition of $\hat{f}$, we clearly have for all $z=(\pi, y)$ and $z^{\prime}=\left(\pi^{\prime}, y^{\prime}\right)$ in $K^{m} \times \mathbb{R}^{d}$,

$$
\begin{aligned}
\left|\hat{f}(z)-\hat{f}\left(z^{\prime}\right)\right| & \leq\|f\|_{\text {sup }}\left|\pi-\pi^{\prime}\right|_{1}+[f]_{l i p}\left|y-y^{\prime}\right|_{1} \\
& \leq \bar{f}\left|z-z^{\prime}\right|_{1},
\end{aligned}
$$

A similar inequality holds for $[h]_{l i p}$ i.e. $[\hat{h}]_{l i p} \leq \bar{h}$. Plugging into (4.8) and using (4.6) yields

$$
\begin{aligned}
{\left[v_{k}\right]_{l i p} \leq \bar{h} } & +\bar{L}_{g}(3 \bar{f}+(n-k)(\bar{f}+\bar{h})) \\
& +2 \bar{L}_{g}\left[v_{k+1}\right]_{l i p} .
\end{aligned}
$$

Since $\left[v_{n}\right]_{l i p}=[\hat{h}]_{l i p}$, a straightforward induction gives (4.7).

\section{Proof of Theorem 4.1.}

We set $\Phi_{k}(z)=\mathbb{E}\left[\hat{f}\left(Z_{k+1}\right)+v_{k+1}\left(Z_{k+1}\right) \mid Z_{k}=z\right]$ and $\hat{\Phi}_{k}(z)=\mathbb{E}\left[\hat{f}\left(\hat{Z}_{k+1}\right)+\hat{v}_{k+1}\left(\hat{Z}_{k+1}\right) \mid \hat{Z}_{k}=\right.$ $z]$. Then, for $k=0, \ldots, n-1$,

$$
\begin{aligned}
\left\|v_{k}\left(Z_{k}\right)-\hat{v}_{k}\left(\hat{Z}_{k}\right)\right\|_{1} \leq & \left\|\hat{h}\left(Z_{k}\right)-\hat{h}\left(\hat{Z}_{k}\right)\right\|_{1}+\left\|\Phi_{k}\left(Z_{k}\right)-\hat{\Phi}_{k}\left(\hat{Z}_{k}\right)\right\|_{1} \\
\leq & {[\hat{h}]_{l i p}\left\|Z_{k}-\hat{Z}_{k}\right\|_{1}+\left\|\Phi_{k}\left(Z_{k}\right)-\Phi_{k}\left(\hat{Z}_{k}\right)\right\|_{1} } \\
& +\left\|\mathbb{E}\left[\Phi_{k}\left(\hat{Z}_{k}\right) \mid \hat{Z}_{k}\right]-\mathbb{E}\left[\Phi_{k}\left(Z_{k}\right) \mid \hat{Z}_{k}\right]\right\|_{1}+\left\|\mathbb{E}\left[\Phi_{k}\left(Z_{k}\right) \mid \hat{Z}_{k}\right]-\hat{\Phi}_{k}\left(\hat{Z}_{k}\right)\right\|_{1} \\
\leq & {[\hat{h}]_{l i p}\left\|Z_{k}-\hat{Z}_{k}\right\|_{1}+2\left\|\Phi_{k}\left(Z_{k}\right)-\Phi_{k}\left(\hat{Z}_{k}\right)\right\|_{1} } \\
& +\left\|\mathbb{E}\left[\Phi_{k}\left(Z_{k}\right) \mid \hat{Z}_{k}\right]-\hat{\Phi}_{k}\left(\hat{Z}_{k}\right)\right\|_{1},
\end{aligned}
$$


by the law of iterated conditional expectation. Since $\hat{Z}_{k}$ is $\sigma\left(Z_{k}\right)$-measurable, we have

$$
\begin{aligned}
& \left\|\mathbb{E}\left[\Phi_{k}\left(Z_{k}\right) \mid \hat{Z}_{k}\right]-\hat{\Phi}_{k}\left(\hat{Z}_{k}\right)\right\|_{1} \\
= & \left\|\mathbb{E}\left[\hat{f}\left(Z_{k+1}\right)+v_{k+1}\left(Z_{k+1}\right) \mid \hat{Z}_{k}\right]-\mathbb{E}\left[\hat{f}\left(\hat{Z}_{k+1}\right)+\hat{v}_{k+1}\left(\hat{Z}_{k+1}\right) \mid \hat{Z}_{k}\right]\right\|_{1} \\
\leq & {[\hat{f}]_{l i p}\left\|Z_{k+1}-\hat{Z}_{k+1}\right\|_{1}+\left\|v_{k+1}\left(Z_{k+1}\right)-\hat{v}_{k+1}\left(\hat{Z}_{k+1}\right)\right\|_{1} . }
\end{aligned}
$$

Plugging into (4.11) yields :

$$
\begin{gathered}
\left\|v_{k}\left(Z_{k}\right)-\hat{v}_{k}\left(\hat{Z}_{k}\right)\right\|_{1} \leq\left([\hat{h}]_{l i p}+2\left[\Phi_{k}\right]_{l i p}\right)\left\|Z_{k}-\hat{Z}_{k}\right\|_{1}+[\hat{f}]_{l i p}\left\|Z_{k+1}-\hat{Z}_{k+1}\right\|_{1} \\
+\left\|v_{k+1}\left(Z_{k+1}\right)-\hat{v}_{k+1}\left(\hat{Z}_{k+1}\right)\right\|_{1} .
\end{gathered}
$$

Since $\left\|v_{n}\left(Z_{n}\right)-\hat{v}_{n}\left(\hat{Z}_{n}\right)\right\|_{1} \leq[\hat{h}]_{l i p}\left\|Z_{n}-\hat{Z}_{n}\right\|_{1}$, a direct induction gives :

$$
\left\|v_{k}\left(Z_{k}\right)-\hat{v}_{k}\left(\hat{Z}_{k}\right)\right\|_{1} \leq \sum_{j=k}^{n} a_{j}\left\|Z_{j}-\hat{Z}_{j}\right\|_{1}
$$

where

$$
a_{j}=\left\{\begin{array}{cc}
{[\hat{h}]_{l i p}+2\left[\Phi_{k}\right]_{l i p},} & j=k \\
{[\hat{h}]_{l i p}+2\left[\Phi_{j}\right]_{l i p}+[\hat{f}]_{l i p},} & j=k+1, \ldots n-1 \\
{[\hat{h}]_{l i p}+[\hat{f}]_{l i p},} & j=n
\end{array}\right.
$$

Now, by Lemmata 3.1 and 4.1, we have

$$
\begin{aligned}
{\left[\Phi_{k}\right]_{l i p}=} & {\left[R_{k+1}\left(\hat{f}+v_{k+1}\right)\right]_{l i p} } \\
\leq & \bar{L}_{g}\left(\|\hat{f}\|_{\text {sup }}+2[\hat{f}]_{l i p}+\left\|v_{k+1}\right\|_{\text {sup }}+2\left[v_{k+1}\right]_{l i p}\right) \\
\leq & \bar{L}_{g}[3 \bar{f}+(n-k)(\bar{f}+\bar{h})] \\
& \quad+(\bar{f}+\bar{h})\left(\frac{n-k}{2}+1\right) \frac{\left(2 \bar{L}_{g}\right)^{n-k+1}}{2 \bar{L}_{g}-1} .
\end{aligned}
$$

Substituting into (4.13) and (4.12) provides the required result by using also CauchySchwarz inequality.

\section{$5 \quad$ Numerical illustration : Bermudean options in a partially observed stochastic volatility model}

We consider an observable risky asset price $\left(S_{k}\right)$ with dynamics given by :

$$
S_{k+1}=S_{k} \exp \left[\left(r-\frac{1}{2} X_{k}^{2}\right) \delta+X_{k} \sqrt{\delta} \varepsilon_{k+1}\right], \quad S_{0}=s_{0}>0
$$

where $\left(\varepsilon_{k}\right)$ is a sequence of Gaussian white noise, and $\left(X_{k}\right)$ is the unobservable volatility process. $\delta>0$ may represent some discretization time step. Equivalently, we observe the 
process $\left(Y_{k}\right)=\left(\ln S_{k}\right)$, and we notice that the conditional law of $Y_{k+1}$ given $\left(X_{k}, Y_{k}\right)$ has a density given by :

$$
g\left(X_{k}, Y_{k}, y^{\prime}\right)=\frac{1}{\sqrt{2 \pi X_{k}^{2} \delta}} \exp \left[-\frac{\left(y^{\prime}-Y_{k}-\left(r-\frac{1}{2} X_{k}^{2}\right) \delta\right)^{2}}{2 X_{k}^{2} \delta}\right], \quad y^{\prime} \in \mathbb{R} .
$$

We model here the dynamics of $(X, S)$ under some risk neutral martingale measure $\mathbb{P}, r$ representing in this case the riskless interest rate.

We assume that $\left(X_{k}\right)$ is an homogeneous Markov chain taking three possible values $x^{b}$ $<x^{m}<x^{h}$ in $\mathbb{R}_{+} \backslash\{0\}$. Its probability transition matrix is given by:

$$
P_{k}=\left(\begin{array}{ccc}
1-\left(p_{b m}+p_{b h}\right) \delta & p_{b m} \delta & p_{b h} \delta \\
p_{m b} \delta & 1-\left(p_{m b}+p_{m h}\right) \delta & p_{m h} \delta \\
p_{h b} \delta & p_{h m} \delta & 1-\left(p_{h b}+p_{h m}\right) \delta
\end{array}\right)
$$

In this context of a partially observed stochastic volatility model, we consider a Bermudean put option with payoff :

$$
h(y)=\left(\kappa-e^{y}\right)_{+}, \quad y \in \mathbb{R},
$$

and we want to compute its price given by :

$$
u_{0}=\sup _{\tau \in \mathcal{T}_{n}^{Y}} \mathbb{E}\left[e^{-r \tau \delta} h\left(Y_{\tau}\right)\right]
$$

We consider a model where the volatility $\left(X_{k}\right)$ is a Markov-chain approximation à la Kushner (see [8]) of a mean-reverting process :

$$
d X_{t}=\lambda\left(x_{0}-X_{t}\right) d t+\eta d W_{t} .
$$

Denoting by $\Delta>0$ the spatial step, this corresponds to a probability transition matrix of the form (5.1) with :

$$
x^{b}=x_{0}-\Delta, \quad x^{m}=x_{0}, \quad x^{h}=x_{0}+\Delta,
$$

and

$$
\begin{aligned}
p_{b m}=\lambda+\frac{\eta^{2}}{2 \Delta^{2}}, & p_{b h}=0 \\
p_{m b}=\frac{\eta^{2}}{2 \Delta^{2}}, & p_{m h}=\frac{\eta^{2}}{2 \Delta^{2}} \\
p_{h b}=0, & p_{h m}=\lambda+\frac{\eta^{2}}{2 \Delta^{2}} .
\end{aligned}
$$

In order to ensure that $P_{k}$ is indeed a probability transition matrix, we have the consistency conditions :

$$
1-\left(\lambda+\frac{\eta^{2}}{2 \Delta^{2}}\right) \delta \geq 0 \quad \text { and } \quad 1-\frac{\eta^{2}}{\Delta^{2}} \delta \geq 0
$$

We perform numerical tests with 
- Price and put option parameters : $r=0.05, S_{0}=110, \kappa=100$,

- Volatility parameters : $\lambda=1, \eta=0,1, X_{0}=0.15$,

- Spatial step : $\Delta=0,05$.

- Quantization : Grids are of same size $N$ fixed for each time period with step $\delta=\frac{1}{n}$.

We first compare in Table 1 the filter expectation at the final date computed with a time step size $\delta=1 / 5$ and by using the optimal quantization method with increasing grid size $N$, and with $10^{6}$ Monte Carlo iterations.

\begin{tabular}{|c|c|c|c|c|}
\hline & $E\left[\Pi_{n}^{1}\right]$ & $E\left[\Pi_{n}^{2}\right]$ & $E\left[\Pi_{n}^{3}\right]$ & Relative error (\%) \\
\hline Monte Carlo & 0.287608 & 0.422833 & 0.289558 & \\
\hline Quant. with $N=300$ & 0.301651 & 0.421725 & 0.276624 & 0.898 \\
\hline Quant. with $N=600$ & 0.301604 & 0.421458 & 0.276938 & 0.886 \\
\hline Quant. with $N=900$ & 0.301598 & 0.421316 & 0.277086 & 0.881 \\
\hline Quant. with $N=1200$ & 0.301618 & 0.42122 & 0.277162 & 0.879 \\
\hline Quant. with $N=1500$ & 0.301605 & 0.421205 & 0.27719 & 0.878 \\
\hline
\end{tabular}

Table 1: Comparison of quantized filter value to its Monte Carlo estimation

We observe that besides the very low error level, the absolute error (plotted in Figure 1) and the relative error are decreasing as the grid size grows.

Secondly, in order to illustrate the effect of the time step, we compute the American option price under partial observation when the time step $\delta$ decreases to zero (i.e. $n$ increases) and compare it with the American option price with complete observation of $\left(X_{k}, Y_{k}\right)$. Indeed, in the limit for $\delta \rightarrow 0$ we fully observe the volatility, and so the partial observation price should converge to the complete observation price.

Moreover, when we have more and more observations, the difference between the two prices should decrease and converge to zero. This is shown in figure 2, where we performed option pricing over grids of size $N_{\Pi, Y}=1500$ in case of partial observation. The total observation price is given by the same pricing algorithm carried out on $N_{X, Y}=45$ points for the product grid of $\left(X_{k}, Y_{k}\right)$. We have seen in Remark 4.1 that for fixed $n$, the rate of convergence for the approximation of the value function under partial observation is of order $N_{\Pi, Y}^{1 /(m-1+d)}$ where $N_{\Pi, Y}$ is the number of points used at each time $k$ for the grid of $\left(\Pi_{k}, Y_{k}\right)$ valued in $K^{m} \times \mathbb{R}^{d}$. From results of [1], we also know that the rate of convergence for the approximation of the value function under full observation is of order $m \times N_{Y}$ where $N_{X, Y}=m \times N_{Y}$ is the number of points at each time $k$, used for the grid of $\left(X_{k}, Y_{k}\right)$ valued in $E \times \mathbb{R}^{d}$. This explains why, in order to have comparable results, and with $m=3$ and $d$ $=1$, we have chosen $N_{Y} \sim N_{\Pi, Y}^{1 / 3}$.

In addition, it is possible to observe the effect of information enrichment as the time step decreases. In fact, if we consider multiples of $n$ as the time step parameter, we notice that the American option price increases for both total and partial observation models (see tables 2 and 3 ). 


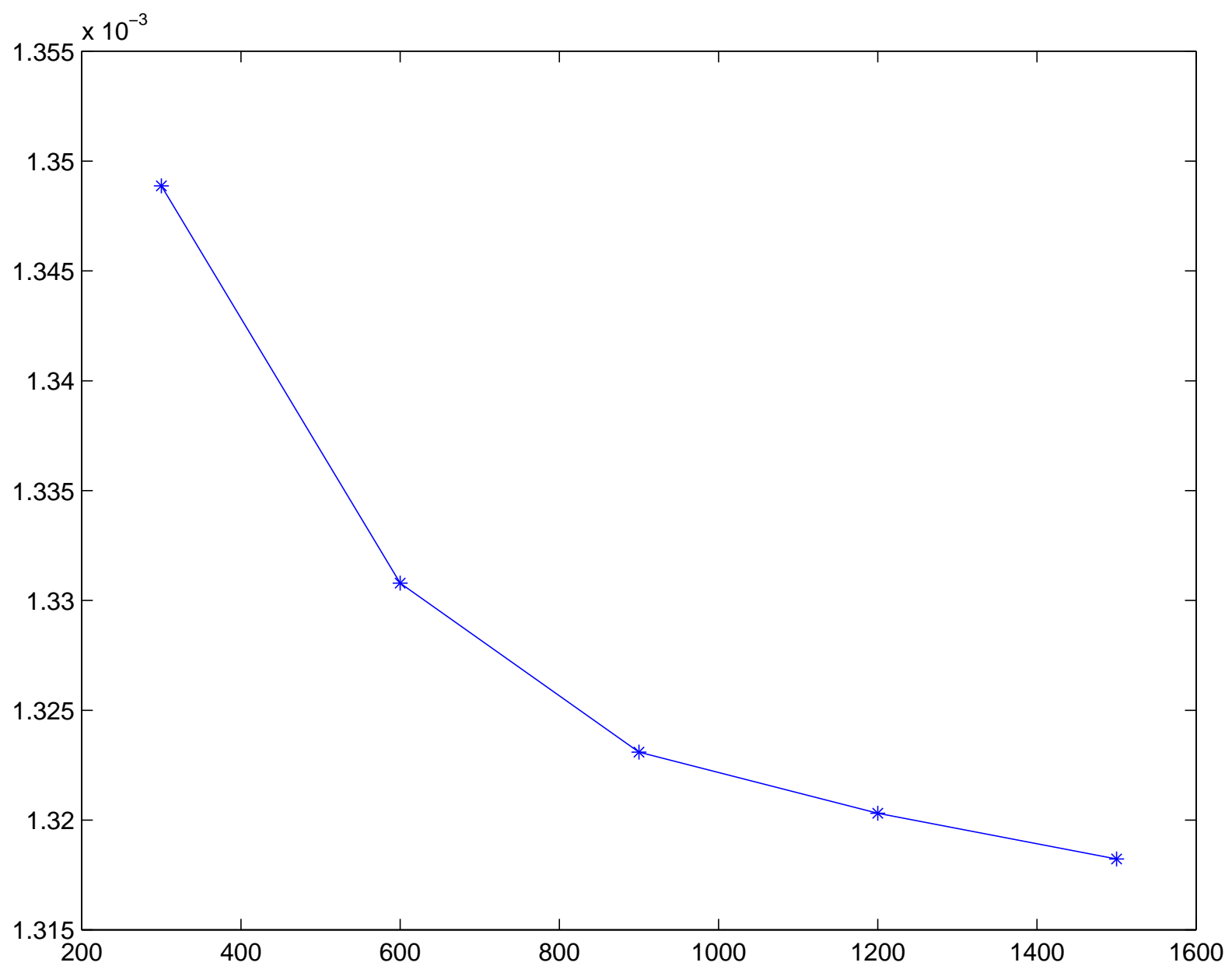

Figure 1: Filter error convergence as N grows

\begin{tabular}{|c|c|c|c|}
\hline$n$ & 4 & 8 & 16 \\
\hline Tot. Obs. $\left(N_{X, Y}=30\right)$ & 1.45863 & 1.75689 & 1.77642 \\
\hline Part. Obs. $\left(N_{\Pi, Y}=1000\right)$ & 0.921729 & 1.13898 & 1.47089 \\
\hline Variation & 0.53 & 0.61 & 0.30 \\
\hline
\end{tabular}

Table 2: American option price for embedded filtrations - First Example

\begin{tabular}{|c|c|c|c|}
\hline$n$ & 5 & 10 & 20 \\
\hline Tot. Obs. $\left(N_{X, Y}=45\right)$ & 1.57506 & 1.72595 & 1.91208 \\
\hline Part. Obs. $\left(N_{\Pi, Y}=1500\right)$ & 0.988531 & 1.30616 & 1.59632 \\
\hline Variation & 0.58 & 0.42 & 0.31 \\
\hline
\end{tabular}

Table 3: American option price for embedded filtrations - Second Example 


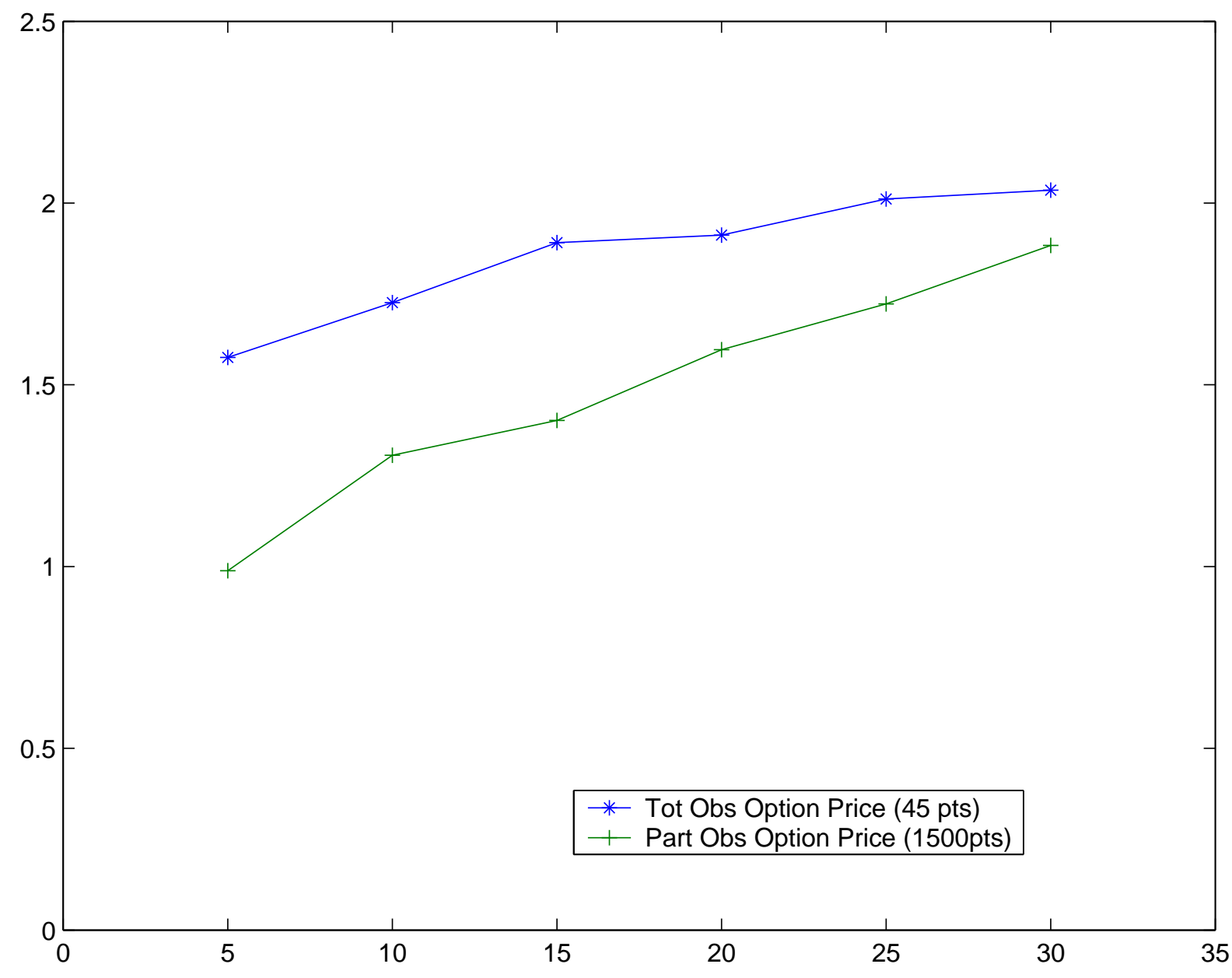

Figure 2: Partial and total observation option prices as $\delta \rightarrow 0$ 


\section{References}

[1] V. Bally, G. Pagès (2003): A quantization algorithm for solving discrete time multi-dimensional optimal stopping problems, Bernoulli, 9, 1003-1049.

[2] Bally V., Pagès G., Printems J. (2001) : A stochastic quantization method for non linear problems, Monte Carlo Methods and Applications, 7, $\mathrm{n}^{0} 1-2$, pp.21-34.

[3] A. Bensoussan and W. Runggaldier (1987) : An approximation method for stochastic control problems with partial observation of the state : a method for constructing $\varepsilon$-optimal controls, Acta Appli. Math., 10, 145-170.

[4] P. Del Moral, J. Jacod, P. Protter (2001) : The Monte-Carlo method for filtering with discretetime observations, Prob. Theory Rel. Fields, 120, 346-368.

[5] Di Masi G.B., Runggaldier W.J. (1987) : An Approach to Discrete-Time Stochastic Control Problems under Partial Observation, SIAM J. Control \& Optimiz. 25, pp. 38 - 48.

[6] Duflo, M. (1997): Random Iterative Models, Coll. Applications of Mathematics, 34, SpringerVerlag, Berlin, 1997, 385p.

[7] Graf S., Luschgy H. (2000): Foundations of quantization for random vectors, Lecture Notes in Mathematics $n^{0} 1730$, Springer, Berlin, 230 pp.

[8] Kushner H.J., Dupuis P.G. (1992) : Numerical Methods for Stochastic Control Problems in Continuous Time, Springer, New York.

[9] Kushner H.J., Yin G.G. (1997): Stochastic Approximation Algorithms and Applications, Springer, New York.

[10] N. Newton (2001) : Approximations for nonlinear filters based on quantization, Monte Carlo Methods and Appl, 7, 311-320.

[11] Pagès G. (1997): A space vector quantization method for numerical integration, Journal of Computational and Applied Mathematics, 89, pp.1-38.

[12] G. Pagès, H. Pham (2003): Optimal quantization methods for nonlinear filtering with discretetime observations, Preprint, Laboratoire de Probabilités et modèles aléatoires, Universités Paris $6 \& 7$ (France).

[13] G. Pagès, H. Pham, J. Printems (2003): An optimal Markovian quantization algorithm for multidimensional stochastic control problems, to appear in Stochastics and Dynamics.

[14] G. Pagès, H. Pham, J. Printems (2003): Optimal quantization methods and applications to numerical problems in finance, to appear in Handbook of computational and numerical methods in finance, ed. S. Rachev, Springer Verlag.

[15] Runggaldier W.J., Stettner E. (1991) : On the Construction of Nearly Optimal Strategies for a General Problem of Control of Partially Observed Diffusions, Stochastics and Stochastics Reports, 37 , pp.15-47.

[16] A. Sellami (2004) : Nonlinear filtering with observation quantization, Preprint, Laboratoire de Probabilités et modèles aléatoires, Universités Paris 6 \& 7 (France). 